February 2021

\title{
Identification of LSB image Steganography using Cover Image Comparisons
}

\author{
Michael Pelosi \\ Texas A\&M University - Texarkana, mpelosi@tamut.edu \\ Chuck Easttom \\ Independent Forensic Expert, chuckeasttom@gmail.com
}

Follow this and additional works at: https://commons.erau.edu/jdfsl

Part of the Computer Law Commons, and the Information Security Commons

\section{Recommended Citation}

Pelosi, Michael and Easttom, Chuck (2021) "Identification of LSB image Steganography using Cover Image Comparisons," Journal of Digital Forensics, Security and Law. Vol. 15 , Article 6.

DOI: https://doi.org/10.15394/jdfsl.2021.1551

Available at: https://commons.erau.edu/jdfsl/vol15/iss2/6

This Article is brought to you for free and open access by the Journals at Scholarly Commons. It has been accepted for inclusion in Journal of Digital Forensics, Security and Law by an authorized administrator of Scholarly Commons. For more information, please contact commons@erau.edu.

(c)ADFSL 


\title{
POSITIVE IDENTIFICATION OF LEAST SIGNIFICANT BIT (LSB) ${ }^{1}$ IMAGE STEGANOGRAPHY USING COVER IMAGE COMPARISONS
}

\begin{abstract}
Steganography has long been used to counter forensic investigation. This use of steganography as an antiforensics technique is becoming more widespread. This requires forensic examiners to have additional tools to more effectively detect steganography. In this paper we introduce a new software concept specifically designed to allow the digital forensics professional to clearly identify and attribute instances of least significant bit (LSB) image steganography by using the original cover image in side-by-side comparison with a suspected steganographic payload image. This technique is embodied in a software implementation named CounterSteg. The CounterSteg software allows detailed analysis and comparison of both the original cover image and any modified image, using sophisticated bit- and color-channel visual depiction graphics. In certain cases, the steganographic software used for message transmission can be identified by the forensic analysis of LSB and other changes in the payload image. This paper demonstrates usage and typical forensic analysis with eight commonly available steganographic programs. Future work will attempt to automate the typical types of analysis and detection. This is important, as currently there is a steep rise in the use of image LSB steganographic techniques to hide the payload code used by malware and viruses, and for the purposes of data exfiltration. This results because of the fact that the hidden code and/or data can more easily bypass virus and malware signature detection in such a manner as being surreptitiously hidden in an otherwise innocuous image file.
\end{abstract}

Keywords: Steganography, steganalysis, digital forensics, malware, virus, LSB encoding.

1 Least Significant Bit (LSB) steganography is a technique which encodes data to the least significant bit of pixel color channel data in an image. This can include the red, green, blue, or alpha color channel data. The LSB is favorable for potential modification since changes here will result in the least detectable visual artifacts to the human viewer compared to the original image. 


\section{INTRODUCTION}

There are multiple uses for steganography, including hiding information and secret communications. Many are meant to disrupt forensic analysis (Walia \& Navdeep, 2010). Steganography use in malware and for covert communications is on the rise according to many computer security research organizations (Sutherland, Davies, \& Blyth, 2011). Payload images can also bypass data exfiltration mechanisms with relative ease, as positive identification of message carrying additional embedded information is elusive.

Steganography has been successfully used for data exfiltration, espionage, concealed communications, command and control for botnets ${ }^{2}$ orchestration, malvertising, and ransomware propagation, among other covert and malicious applications. Below is a list of examples how steganography can typically operate in various application case scenarios (Gutub, Ankeer, Abu-Ghalioun, Shaheen, \& Alvi 2008):

- An employee decides to steal sensitive proprietary files. With today's security systems, this would be noticed using classic approaches, such as unauthorized downloads detected in a logging system or firewall filter. However, using steganography, the sensitive files are encoded into images. By doing so, the images can be uploaded to social networks or cloud storage services without triggering red flags, such as additional copyright or intellectual property checks (many of these are automated using machine learning systems on the larger social media platforms such as YouTube, Facebook, Instagram, and Twitter). Additionally, original sensitive files, such as business plans, accounting records, patent applications, or movie scripts, would appear suspect and trigger red flag sensations in even casual laypersons browsing such content.

- A group of cybercriminals is attempting to communicate and synchronize attacks from different countries. Since they cannot go through standard communication channels ${ }^{3}$, they decide to conceal secret messages into profile pictures of social accounts. In this way, they can communicate by uploading and downloading unsuspicious profile photos using whitelisted services.

- A global botnet with over one million active bots has been deployed and is awaiting instructions. Any attempt of communication from a central server to the bots is likely to be discovered eventually, due to massive redundant and duplicated packets traceable back to the C\&C nodes and finally, the root BotMaster. Instead of using a server, the BotMaster has the engineered the bots as configured to periodically download a feed of text and images from a public social network account. By decoding steganographic data from the feed, instructions are extracted and executed more surreptitiously.

- A malicious campaign is planned to affect millions of users but the perpetrators want to keep it as secret as possible. Since the goal is to exploit a browser vulnerability, they use steganography to conceal malicious code into advertisement images. Much simpler Javascript code in the webpages

2 Botnets are collections of compromised computers (Bots) which are remotely controlled by the botnet originator (BotMaster) under a common commond-and-control (C\&C) infrastructure. The $\mathrm{C} \& \mathrm{C}$ is used to distribute commands to the Bots for malicious activities such as distributed denial-of-service (DDoS) attacks, sending large amount of SPAM, and other nefarious purposes (Zeidanloo \& Manal, 2010).

3 Various reasons exist why dubious actors may be hesitant and not capable of using typical public communication channels such as Gmail, among others. First, if one is suspected or investigated it will uncover the entire group due to the networked chains-of-communications. Second, due to the likely conversation content, keyword flags may quickly bring their activities to the attention of authorities. Third, individual actors may lack the valid credentials to create fake accounts not traceable their legal names or entities - such as debit card, mailing address, permanent email or telephone number, or Internet login IP address, necessary to create additional, fake accounts. 
accessed extracts the malicious code and executes it using a technique similar to the one described on the SentinalOne Blog $(2020)^{4}$. To reach a large audience quickly, they submit the banner to networks that distribute the image over hundreds of websites. By doing so the propagation is guaranteed and the campaign revenue can be larger.

- A ransomware attack hides the communication between the victims and the perpetrator. Using steganography information harvested from the target system is encoded into pictures uploaded to an image hosting website. Using this tactic the ransomware campaign deployment can remain hidden for a longer period of time. This is because it will be much more difficult for authorities to determine where the attacks are originating from and where the data is destined for.

The specific examples stated listed are not hypothetical, but rather are based on actual malware case histories (Gutub, Ankeer, Abu-Ghalioun, Shaheen, \& Alvi 2008). Although many of these attacks were eventually identified and stopped, the amount of time and effort required to detect and stop steganography related attacks and communications was considerable and continues to be take investigative resources and knowledge. The result is that steganography continues to be a very lucrative technique and opportunity for cybercriminals.

Regardless of the reasons for employing steganography, there seem to be a finite number of methodologies so far developed for implementing steganography, but new processes are emerging. Least Significant Bit and Discrete Cosine Transform are the two most common methods (Easttom, 2018; Walia, Jain, \& Navdeep, 2010). Therefore, a focus on methods and processes for detecting one or both of these is useful for forensic examiners.

\subsection{Rise in the Usage of Steganography for Malware}

Recently, steganography has been used in the following malware programs and cyberespionage tools (Enterprise Times UK, 2017), (Kaspersky Labs, 2017):

- Microcin, alias Six Little Monkeys;

- NetTraveler;

- Zberp;

- Enfal, which posseses a new loader called Zero.T;

- Shamoon;

- KinS;

- ZeusVM;

- Triton, alias Fibbit.

It is important to understand the causes for this increase in the use of steganography due to the increasing frequency and severity of attacks. There are three primary reasons for this increase. The first is that these methods aid malicious actors in concealing not only the data itself but the fact that data is being uploaded and downloaded (Swain \& Lenka, 2012). A second reason is that steganography can bypass deep packet inspection (DPI) systems (Swain \& Lenka, 2012). A third reason is that the use of steganography may help bypass security checks by anti-APT products (Priyanka, Sahoo 2016; Chen, Huygens, Desmet, \& Joosen, 2016; Daryabar, Dehghantanha, \& Broujerdi, 2011)

It has been confirmed that Steganography was also used by the malware Vawtrak, Zbot, Lurk, and Stegoloader (Heidleburg, 2016; Pevný, Kopp, Křoustek, \& Ker, 2016). In early 2015, Vawtrak started

4 https://www.sentinelone.com/blog/hiding-code-inside-images-malware-steganography/ 
using steganography to hide its settings in favicons. The malware downloads a favicon.ico file from a server hosted on TOR using the tor 2 web service. This favicon.ico image is the one displayed by browsers at the left side of a URL. Generally, each website contains a favicon.ico image, so security products seeing such requests would typically not test them for validity. Next, the malware extracts ${ }^{5}$ a least significant bit from each pixel and constructs a URL for downloading its configuration file. (Wyke, 2015; Pevny, Kopp, Kfoustek, Ker, 2016)

One variant of the Zbot malware also uses steganography to hide its configuration data (Mazurczyk \& Caviglione, 2015). This variant downloads a JPEG onto the victim's system. The configuration data hides inside this image. Later, the malware extracts the configuration data from the image and performs further malicious actions. The configuration data, in particular, must be camouflaged as it may include $\mathrm{C} \& \mathrm{C}$ communications information, such as IP address and port, as well as available commands.

The Lurk malware uses steganography to download other malware onto targeted systems (Heidleburg, 2016). Instead of downloading and executing a malicious binary, Lurk first downloads a BMP image. It uses a least-significant-bit (LSB) algorithm to embed encrypted URLs into the image file. It then extracts the embedded URLs from the image file and then downloads additional malware.

The Stegoloader malware installs malware on victims' systems to steal sensitive information. On successful execution, Stegoloader downloads a PNG image from a legitimate website. It uses steganography to embed its main module's code inside the downloaded PNG. The malware retrieves the hidden data by applying a steganographic extraction algorithm. (Bureau \& Deitrich, 2015)

More recently the Stegano (also known as Astrum) exploit kit, has been used in the past months as part of a very ingenious malvertising campaign. Stegano authors have operated by embedding malicious code inside the RGBA transparency value of each pixel of PNG banner ads. As users viewed the ads, JavaScript code would parse the PNG image, extract the malicious code and redirect the user to the exploit kit landing page, where he would be infected with various types of malware (Daryabar, Dehghantanha \& Broujerdi, 2019).

An exploit kit discovered in 2016 that relies on steganography is named DNSChanger (Cabaj, et al., 2018). The group behind DNSChanger created malicious ads that contained code that launched brute-force attacks against the user's home WiFi router. Attackers were taking control over the victim's router, and injecting ads in all his web traffic. Once again, steganography was crucial to hide this malicious code inside the ads' images, which helped the cybercriminal authors hide the exploit kit's activity from security researchers (Zhang \& Tang, 2018).

One of the major players operating in the exploit kit market has also turned its efforts to using steganography. The exploit kit's name is Sundown, an exploit kit developed a group of German-speaking developers who called themselves the "Yugoslav Business Network" (or YBN) (Zhang \& Tang, 2018).

Until recently, Sundown operators did not mask exploit code delivered to user files. Security researchers looking at traffic logs could easily identify the Sundown exploit package by looking at URLs, which often contained files ending in ".SWF" or ".XAP" extensions, specific to Flash and Silverlight exploits (Daryabar, Dehghantanha \& Broujerdi, 2019).

After a recent update, Sundown now hides these exploits as mundane ".PNG" files. The file's header says the file is a PNG image, but its content contains the actual exploit. Sundown traffic is now much harder to

5 For more details on the typical malware extraction process described, see the following ZDNet article: https://www.zdnet.com/article/hackers-hide-web-skimmer-behind-a-websites-favicon 
detect, and researchers have to put more work in unmasking Sundown operations, just as its operators wanted (Wyke, 2018).

This addition of steganography in Sundown operations was spotted recently and appears to have been inspired by previous three malvertising campaigns:

- The AdGholas malvertising campaign, which ran on the Angler and Neutrino exploit kits.

- The GooNky malvertising campaign.

- The malvertising campaign that delivered the CryLocker ransomware via the RIG exploit kit (Cabaj, et al., 2018).

In the cases cited, the cybercriminals behind these malvertising campaigns had used steganography to deliver PNG images to victims, which contained malicious code that scanned their computer, and later delivered downloaded malware (Wyke, 2018).

The most successful of the malware ad campaigns was the AdGholas campaign, which raged on undetected for almost a year. (Cabaj, et al., 2018) The success of those campaigns has apparently convinced the Sundown organization to run more steganography experiments of its own.

By disguising malicious content as PNG files, Sundown is now following the new trend that has slowly taken hold of the exploit kit market in the past year. It is estimated that it will continue to use steganography, at least until security firms find a way to quickly identify malicious PNG files and block them (Melenson, 2017).

Data exfiltration, also known as data theft, is the unauthorized transfer of sensitive information from a computer or a server (Easttom, 2018). In 2016, there were attacks related to Magento, an online ecommerce platform (Easttom, 2018). The attacks used image steganography to hide payment card details. After execution, the malicious code collected the payment card details and hid this inside a local image file, such as an actual product picture (Easttom, 2018). Once data collection was completed, the attacker then downloaded the image file (typical for an e-commerce website) and extracted the hidden data (Melanson, 2017)

These trends described above in particular suggest that malware writers are on the verge of adopting steganography on a mass scale. Most modern anti-malware solutions provide little, if any, protection from steganography (Pevný, Kopp, Křoustek, \& Ker, 2016). As a result, any steganographic carrier file such as a digital image or even a video file, that can be used to conceal stolen data, or communications between a malware program and a command and control server, will remain undetected.

\subsection{Improved Detection Procedures And Techniques}

Dozens to hundreds of statistical techniques have been developed over the years to attribute a probability of a file being a steganographic cover file but owing to the various methodologies and data payload densities, these methods can be considered unreliable at best (Easttom, 2017). In certain cases, regarding digital image steganography, it may be possible to determine the original image visually or algorithmically. This would be the image file before payload injection takes place.

Normally, image steganography will involve altering the least significant bits (LSBs) in the cover image (Easttom, 2018). By comparing the original image LSBs to the payload image LSBs, a positive identification cannot take place indicating the use of steganography. In performing steganalysis, possessing the payload image file alone leaves little option than the use of statistically based probabilistic tools for attribution. (Walia, Jain, \& Navdeep, 2010) 
However, by locating the original digital image file for comparison, the alteration of LSBs alone is quite the forensic "smoking gun". This "smoking gun" can results in robust and reliable attribution for the use of steganography software to send messages or data files. This could be a starting point for further investigation for law enforcement or other investigative authorities.

In performing sound steganographic procedure, a suspect will take care to data wipe any original cover file, to prevent such a comparison from taking place. However, in practice human error and technical limitations may prevent completely effective data erasure of the original cover image (Melanson, 2017). In that light, we recommend an active search for the original image if suspicion of steganographic usage exists. There are a number of possibilities for locating the original image that will allow later positive attribution for the use of steganography. The following lists many examples of the large number of potential locations to find an original image for comparison. 
Places to find non-payload (also known as a "cover") image (Priyanka \& Sahoo, 2016):

1. Suspect hard drive filesystems

2. Suspect removable USB drives

3. Suspect cameras and mobile devices

4. Suspect CDs and DVDs

5. Local email inboxes/outboxes

6. Cloud email inboxes/outboxes

7. Recent web search and browser histories

8. Google image searches

9. Network attached storage devices

10. Employment computers and networks

11. Recycle bins

12. Deleted files removed from recycle bins

13. Online photo galleries

14. Personal and business associates' files as listed above

Any image presenting the same visual appearance and pixel dimensions is a sound candidate to be the original cover image file. In that light we offer novel digital forensics software to allow the investigator to complete a quick and more convenient LSB analysis in comparison to make a positive identification. Further, the LSB comparison image results can be conveniently copied and pasted for reports documents for conclusive proof of the use of steganography.

\section{THE "COUNTERSTEG" SOFTWARE}

The software introduced with this paper, CounterSteg, is available free of charge from the following website: http://199.175.52.196/CounterSteg/. This Windows-based software allows the loading of two images and comparison of pixel color bits in the LSB plane. It should be noted that this software will also run under Linux with Wine installed. Detailed analysis is performed visually at the moment, however further research is being conducted by the authors on algorithms which can automate the results conclusion positively. The algorithms referenced would attempt to differentiate image changes due to typical image filtering (for example, brightening, contrast enhancement, gamma correction, and sharpening) versus the changes introduced by steganographic activities typically in the LSB plane of pixels.

The CounterSteg software can be used to visually detect differences between original and payload image. Positive detection implies, in general, identical pixel dimensions and most pixels identical except for various LSB values. Human forensic analysis does confirm final analysis using additional informed investigation techniques described in this paper.

\subsection{Recommended Usage}

If a suspect image is detected, a search should be conducted for visually similar and pixel dimensionally identical images in the locations listed above, among others. Visually similar images will differ in file hash values. However, once the original cover image is discovered additional copies of the original image residing on file systems may be identified using hash values. Once potential matches are identified, the software should be used to look for differences in the LSB and perhaps nearby planes. If such differences are detected, it can be considered positive identification for the use of steganography, although it is unlikely the original message or data can be recovered, except with the cooperation of the suspect, or acquisition of the original software and/or encryption key used to embed the data. This is because any data would have to be reconstructed at the individual bit level, for example reassembling ASCII codes for text. This would be 
tedious at best and implausible at worst, and the time resources required would be burdensome. In fact, the clear text message or data may reside elsewhere on the user machine and offer a much lower hanging fruit. However, with positive results for steganography in hand, this further investigation or warrant acquisition can be embarked upon with great confidence.

\subsection{Results Using Various Steganographic Programs for Experimentation and Analysis}

The following research (pages 13-30) outlines the results of steganography analysis and detection with commonly available tools that may be used by a malicious actor. These where chosen because they are available at no charge, and are widely known. These programs include:

- OpenPuff - http://embeddedsw.net/OpenPuff_Steganography_Home.html

- Steganography Online - http://stylesuxx.github.io/steganography/

- Geocaching Toolbox - https://www.geocachingtoolbox.com/index.php?page=steganography

- OTP-Steg - http://www.mauisolarsoftware.com/OTP-Steg/

- f5stego.js - http://desudesutalk.github.io/f5stegojs/

- DevFarmSteganography - https://devfarm.it/steganography/

- StegoShare - http://stegoshare.sourceforge.net/

- BitCrypt - http://bitcrypt.moshe-szweizer.com/

- OpenStego - https://www.openstego.com/index.html

Other programs unable to be tested at this time, but which would be candidates for future study were:

- $\quad$ Steghide - http://steghide.sourceforge.net/download.php

- SteganPEG - http://download.cnet.com/SteganPEG/3000-2193_4-75914262.html

- ManyTools Steganography - https://manytools.org/hacker-tools/steganography-encode-text-intoimage/

- Steganographic Encoder (Steghide) - https://futureboy.us/stegano/encinput.html

- Mobilefish - https://www.mobilefish.com/services/steganography/steganography.php

- Kwebbel - http://www.kwebbel.net/stega/enindex.php

As examples of the type of forensic steganalysis that can be conducted with CounterSteg, the authors of this study have embedded text data into a standard cover image using some of the above listed and easily available steganographic programs. Each of these programs was accessed directly from a website or downloaded executable. Each took less than 10-20 minutes to use to embed the standard text data into the standard cover image, which is shown on the next page in Figure 1.

The standard cover image shown below was taken by one of the authors in Keyser, West Virginia in the Fall of 2015 using a Nikon D90 digital camera. This image shows a fairly even distribution of red, green, and blue colors throughout the image, except for the center top open to the sky. In this specific area, the camera CMOS sensor saturated to white $(\operatorname{RGB}(255,255,255))$, and each of the pixels here represents that single saturated white color. This is notable for LSB steganograpy in that steganographic programs that modify pixels in this area will be more easily statistically detected. Alterations to the LSB values in pixels in solid color, or saturated, portions of the image are a good indicator of nonstandard modifications (such as steganography). Good steganographic programs will attempt to avoid modifications to these specific areas, among others. 


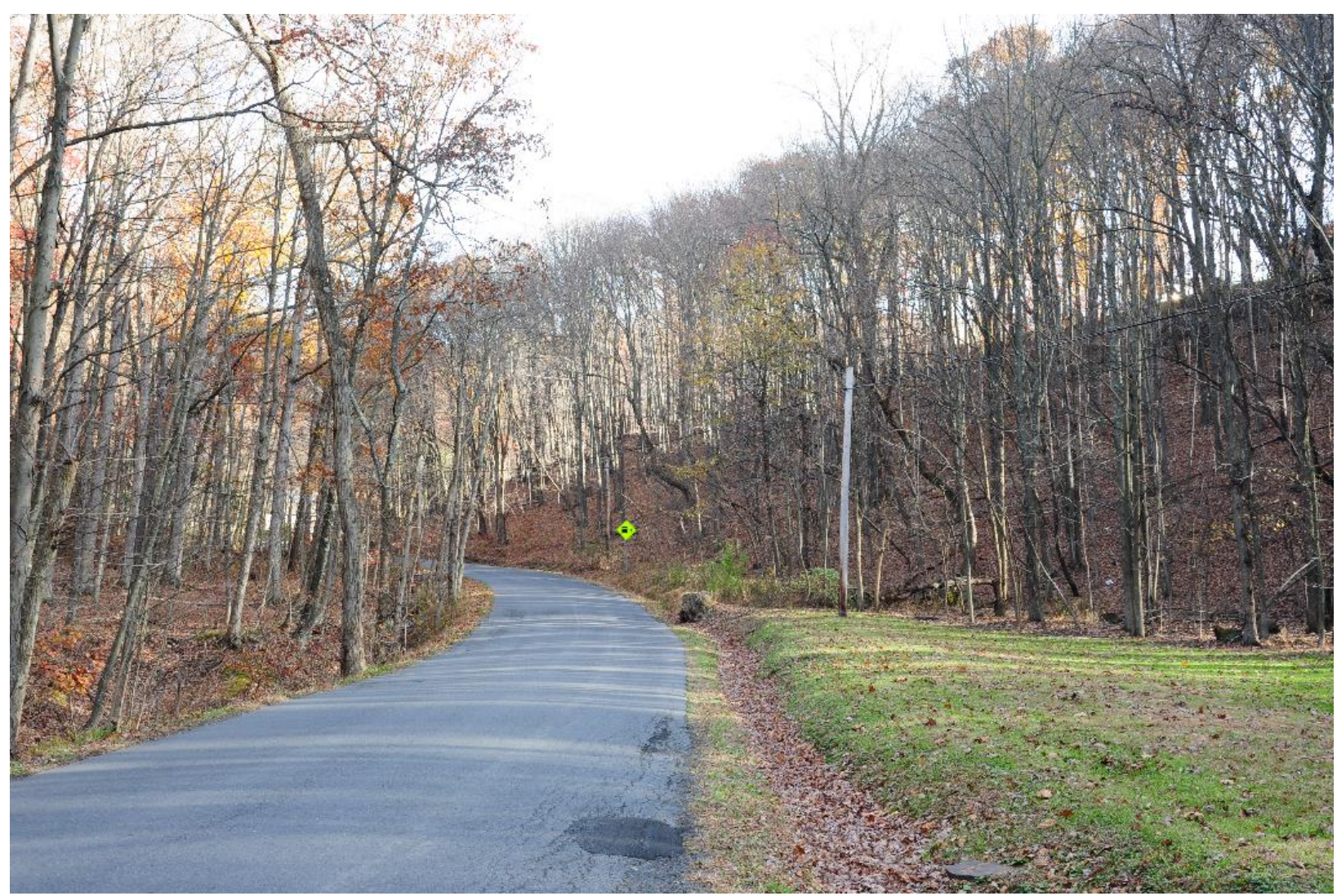

Figure 1. Cover image taken with Nikon D90 camera.

The standard text embedded in the image was the President Kennedy inauguration speech, which is 1,366 words, and 7,512 characters. The size of the text is $7.38 \mathrm{~KB}$ (7,566 bytes), file size on disk was $8.00 \mathrm{~KB}$ (8,192 bytes). Kennedy's inauguration speech was delivered on January 20, 1961.

The CounterSteg program produces detailed visual analysis and comparison of digital images, specifically in each color and bit-plane. In addition to combinations of colors in a particular bit-plane. The figure below shows that the image analysis window, which calculates results for 45 various bit plane and color combinations. The Alpha channel is the transparency channel and remains on unused in many images.

Surprisingly, however, some steganographic programs make spurious or data-carrying modifications in the alpha channel, so it is important to also keep an investigative eye on this color channel (Sutherland, Davies \& Blyth, 2011). In the analysis window shown below, each color channel is broken down by bits, with bit 0 corresponding to the LSB, and bit 7 corresponding to the MSB. The values in each bit plane are shown for red, green, and blue channels, as well as the alpha channel. For the graphic shown for "All Bits", the pixel color here will be non-black if any of the bits (0-7) is set to a nonzero. The color value is the relative intensity of that color (red, green, or blue) in the range of 0 to 255 .

Finally, the image shown in the grid in the upper-left for "All Bits" and "All Colors" is basically the original image, since it shows the combined color values in all channels and all bits. Any of the images shown in the grid can be clicked on to bring up a new window showing that image full-size. This can be copied and pasted into an image editing program for further analysis or the saving of the image.

The overall idea and philosophy of the CounterSteg steganalysis software is to allow the convenient analysis and comparison of before and after images to look for the telltale traces of steganographic software 
activity and modifications. In many cases these follow similar patterns, and the forensic analysis conducted can make informed conclusions based on typical similar patterns from the various categories of steganographic software currently available. The software available generally falls into several categories, which for the purposes of this paper we will categorize as: 3 . the good, 2. the bad, and 1. the ugly.

We will start showing telltale traces from "ugly" steganographic software, typically this quality of software can be easily detected even without the original cover image for comparison. Even in the case of good steganographic software, having the original cover image on hand makes positive identification of the activity highly probable.

The cover image analysis window shown below clearly depicts the area of white saturation in the upper center of the image (the area open to the sky through the trees). Other color and blue channels show a reasonable distribution of intensities throughout most areas of the image. Ideally for steganograhic activities areas of solid colors, saturation, and low noise between colors and shades should be avoided. This is to circumvent statistical analysis of the steganographic payload carrying image that may indicate a high probability of data carrying modifications.

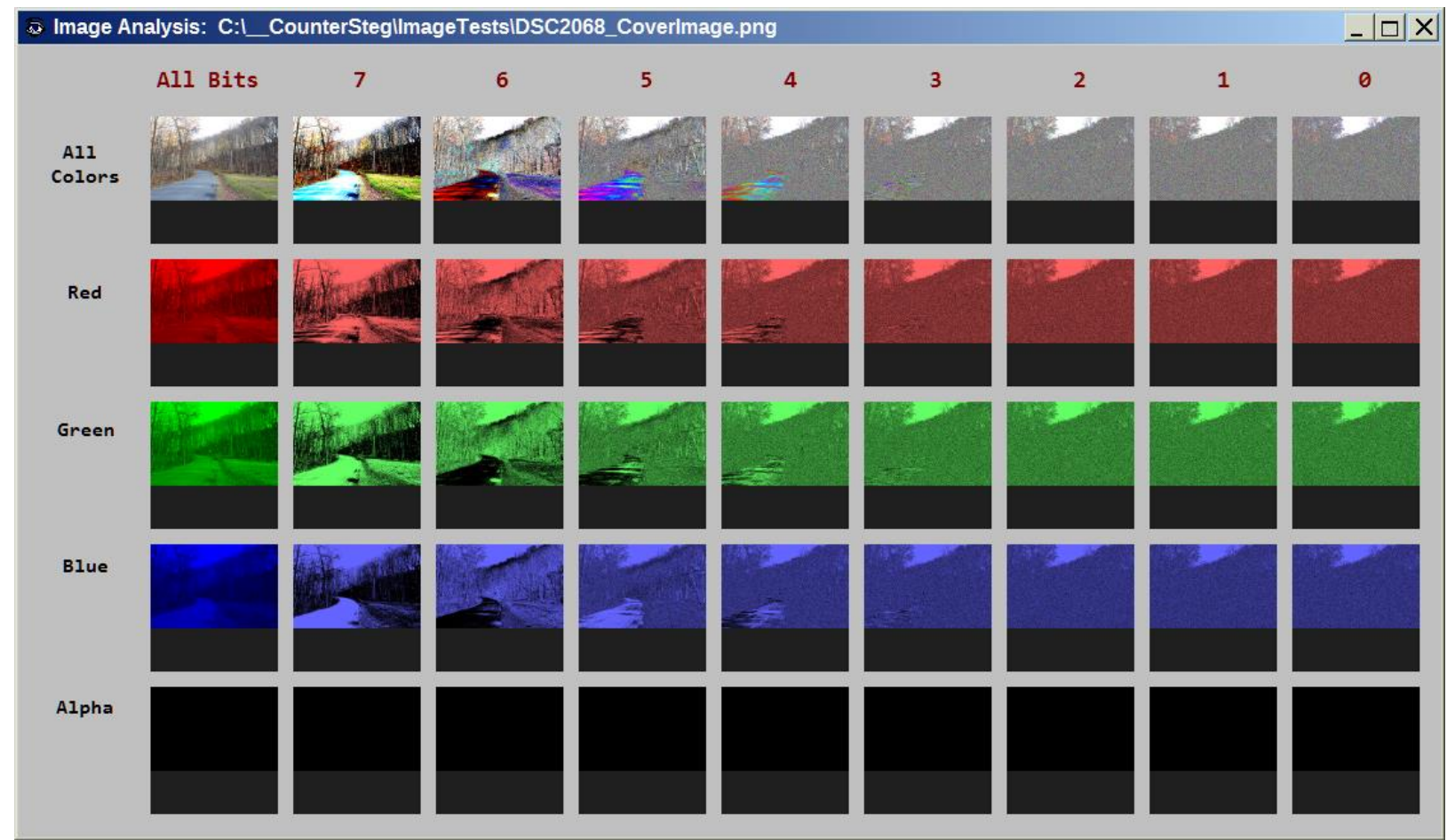

Figure 2. Analysis of cover image.

Clicking on the LSB (bit 0) image for all colors, the image below is brought up and is shown as Figure 3. This image shows the bits colored for whether red, green, or blue pixel LSBs are sent to 0 or 1. Various color shades are shown depending on multiple values, however if only one bit is set, such as red, the pixel will remain red as shown in the red bit 0 color image shown as part of Figure 1. Using the LSB image can give an idea of the relative distribution of LSB values in the image in various colors. In general, for many photographs the distribution will be largely random except for saturated or solid color areas of the image. This image is the starting point for our further analysis, as modifications to the LSB bit plane are particularly evident in certain qualities of steganographic software available (Bhattacharyya,2011). 


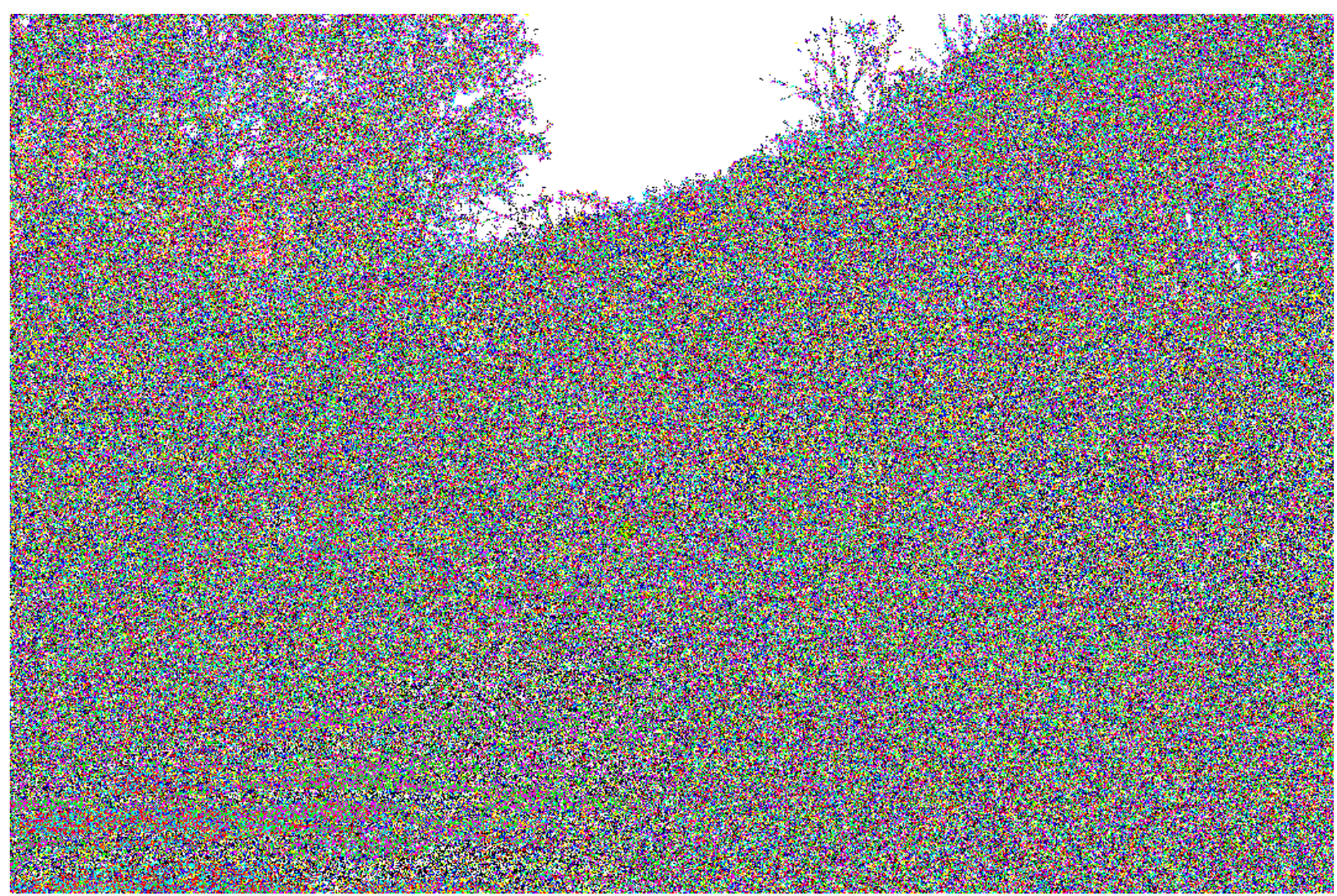

Figure 3. LSB values analysis of cover image.

Also contained within the CounterSteg is the ability to visually show pixel variations between colors in a local area. Below in Figure 4 is an image that shows the variation in the green color channel. Areas of lighter colors indicate higher variation (which could be considered noise) between pixel colors in nearby areas of the image. Since the area of the sky is black — this indicates no variation and LSB, or other bit planes - modifications will be more easily statistically detectable here. Another potentially fruitful area for investigation and comparison is image EXIF data, which can serve as a confirmation of authenticity of certain changes, however this avenue is not explored further in the present research. 
The figure below depicts the green color channel variation using all bits. Variation is based on the calculation of peak signal to noise ratio (PSNR) of each pixel green color versus its neighbors.

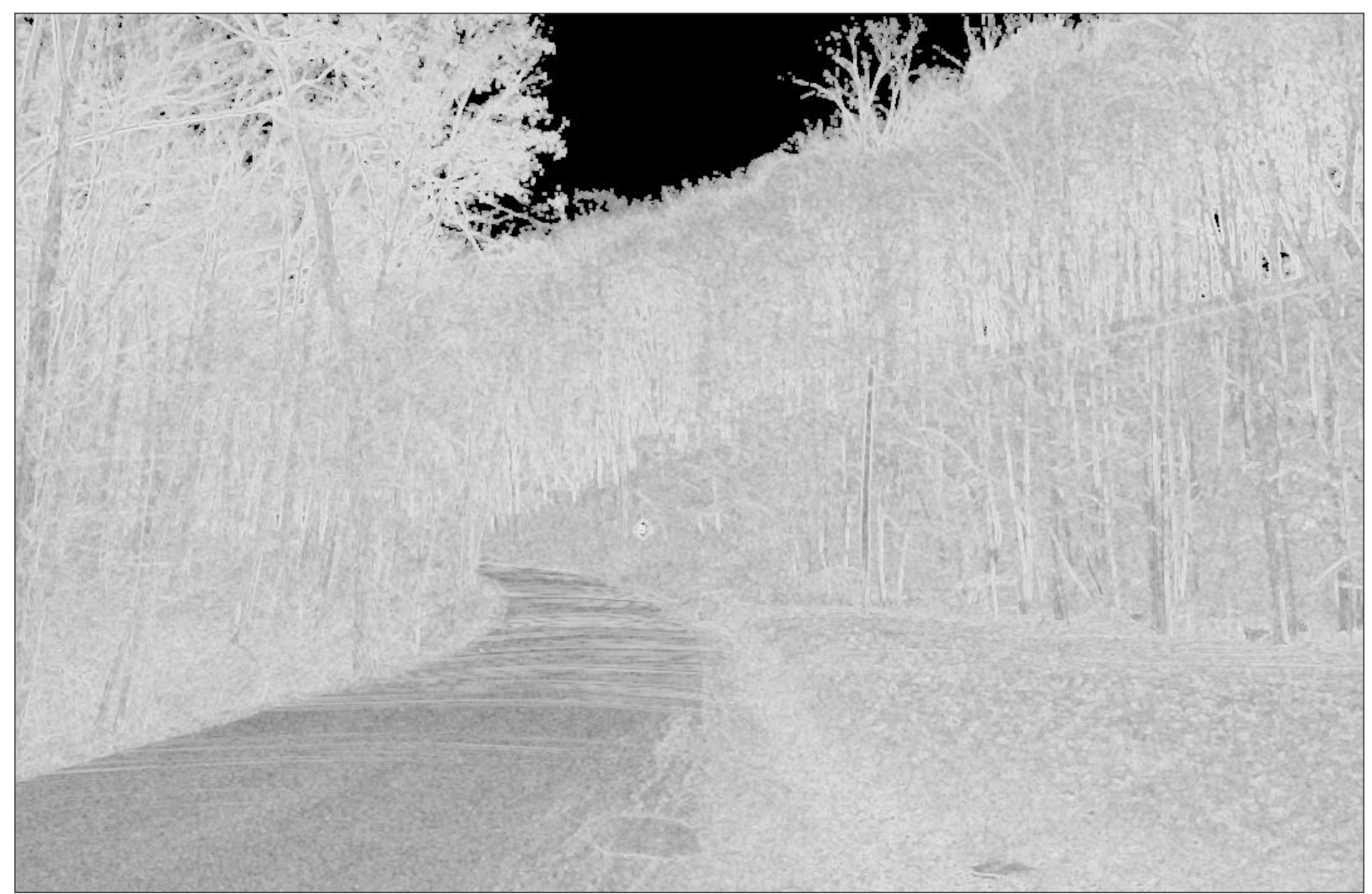

Figure 4. Pixel green color variation throughout the cover image.

In addition to CounterSteg providing bit by bit and color analysis for a single image, the software also allows image comparisons using a similar breakdown. The comparison of the original cover image to a contrast adjusted image is shown below. In this breakdown, only differences between pixels, colors, and bits are shown. Below each comparison image is shown the total number of bit, color, and/or pixel differences (depending on the analysis), as well as the percentage of changes relative to the total number of changes possible.

This analysis window allows quick and convenient comparison between an assumed original cover image, and the assumed steganographic payload image. The specific type, location, and scale of the differences can help to clearly identify steganographic activities that have been performed on the image, the likely image payload size, and perhaps even the likely specific steganographic software that has been used in certain cases. In the following narrative we will detail forensic profiles of various software packages and their results. 


\subsection{Contrast Adjustment}

As an example of a standard image adjustment in comparison, below is the results of comparing the original cover image to a modified image with a small contrast adjustment. In addition to large modifications in the LSB plane we are also seeing large to small modifications in all other bit planes, and in all colors. In general, if two visually similar versions of an image exist - seeing changes like this in comparison would generally not indicate steganographic activities.

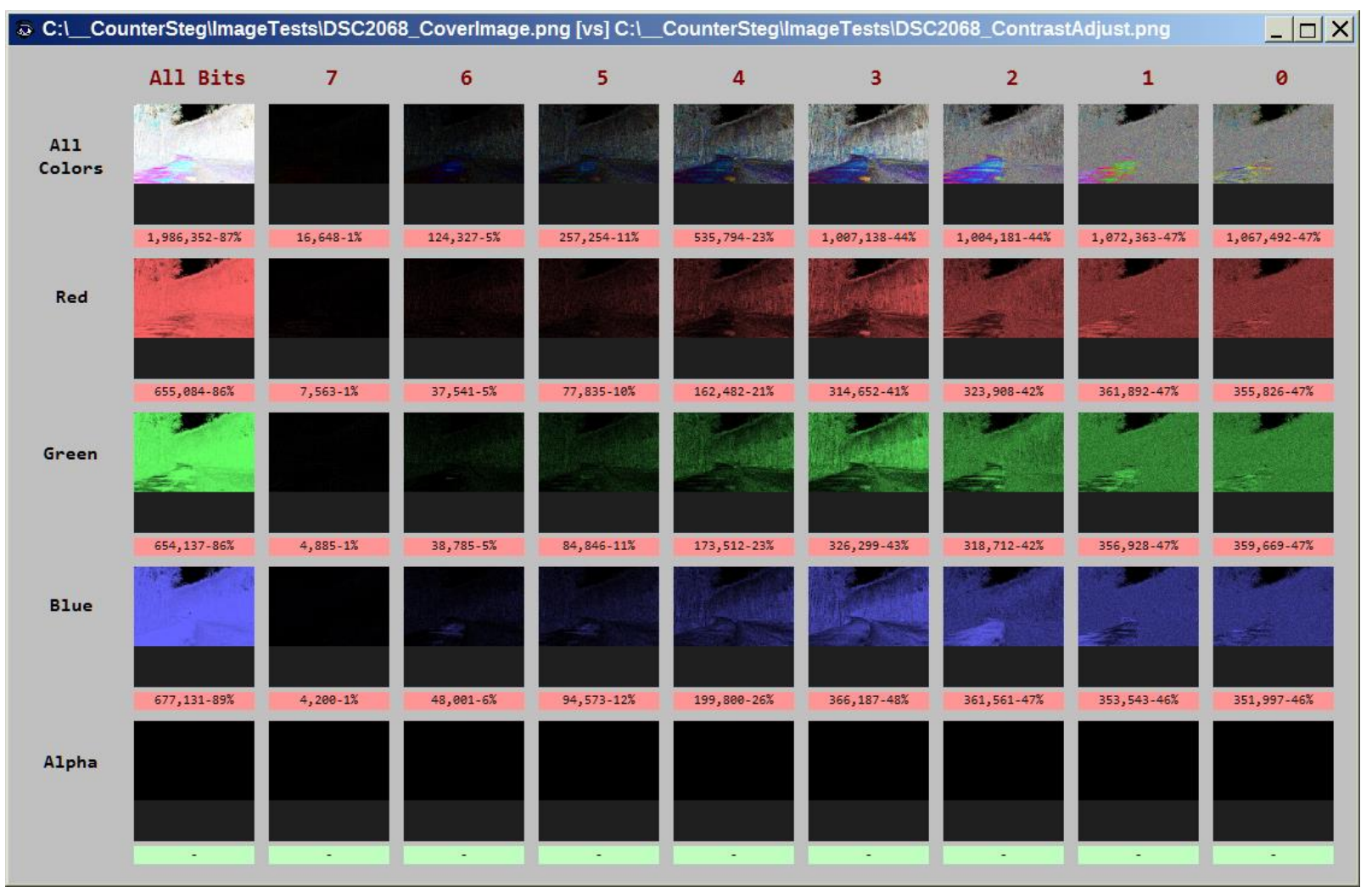

Figure 5. Comparison of cover image to contrast adjusted image. 


\subsection{Brightness Adjustment}

Similar to the small contrast adjustment, a brightness adjustment of the original image results in a comparison profile showing large modifications in all colors and in all bit planes.

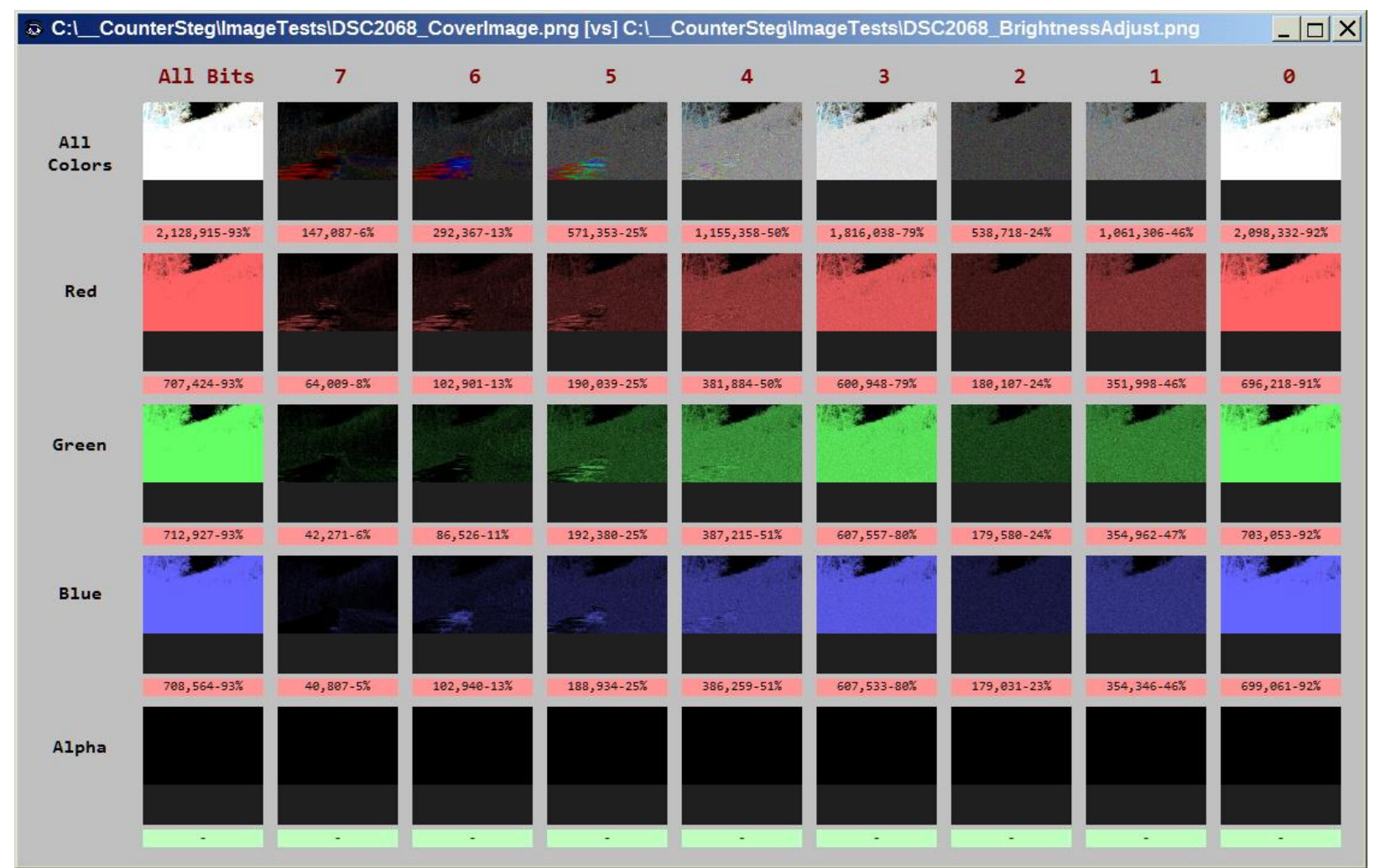

Figure 6. Comparison of cover image to brightness adjusted image. 


\subsection{Gamma Adjustment}

The Gamma adjustment color filter compresses or stretches various colors and would result in a comparison profile similar to the one shown below. All colors and all bit planes are greatly modified. The differences between the two images will be visually apparent.

In conclusion - standard image filters, such as those found in Photoshop, or other image editing software, for contrast, brightness, and gamma adjustment, do not generally yield comparison results similar to what we will depict in the following narrative for steganogrphic related changes. This is with one exception BitCrypt — which should still be detectable using other digital forensic clues and analysis.

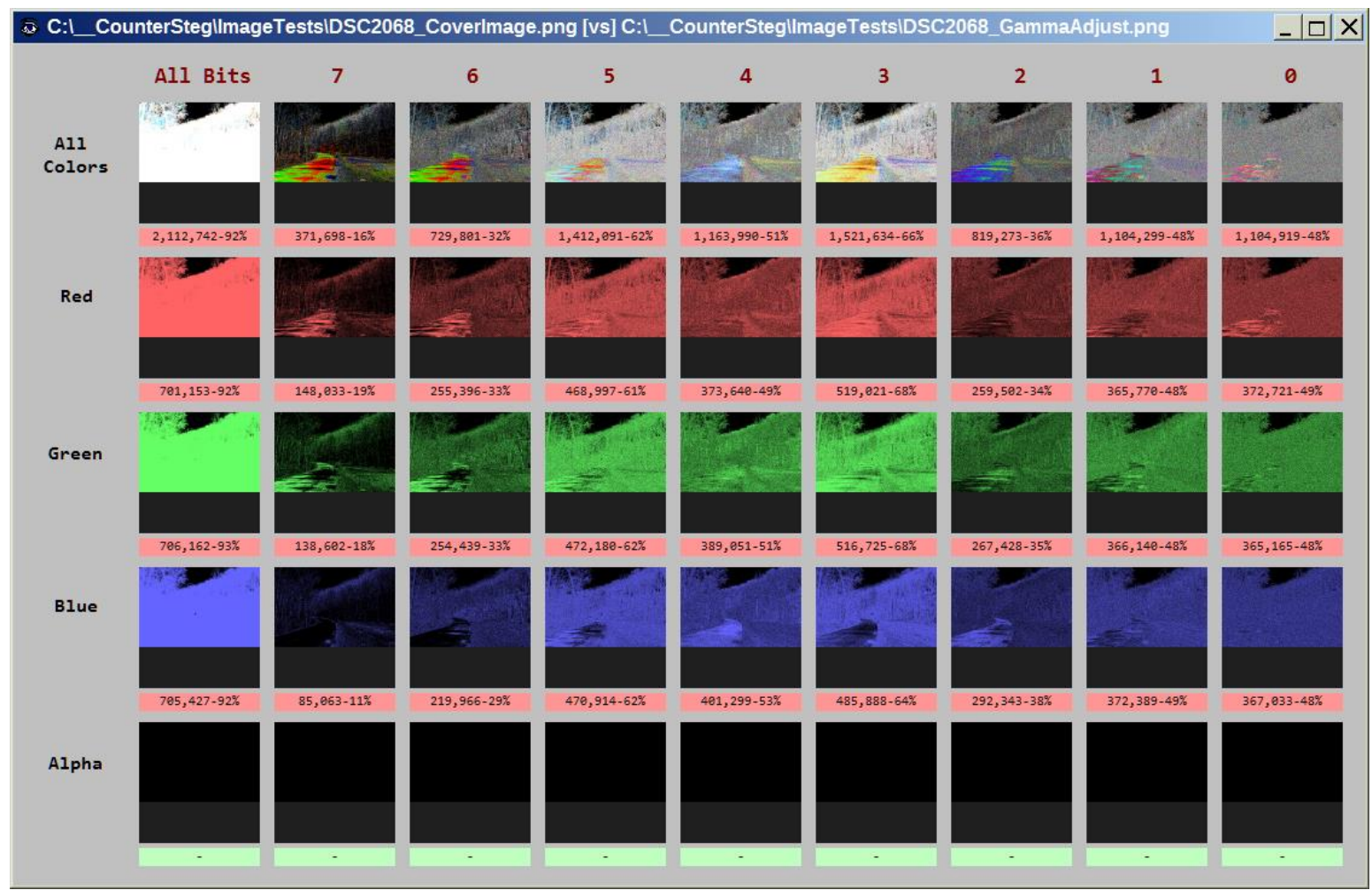

Figure 7. Comparison of cover image to gamma adjusted image.

For the purposes of this paper and analysis, we will divide steganographic software into three general categories ranging from low quality, mid-range quality, and high quality. Each software embeds a digital payload with varying levels of detectable qualities. The low quality provides the least effective steganography, and should result in the easiest forensic detections, even without possessing the original cover image. In the case of high quality steganography software, embedded data will be virtually undetectable without the original cover image for comparison.

However, in the case of high quality steganography software, with the cover image original on hand, a forensic bit plane comparison makes the activity even then easily evident. This is in comparison to typical image changes shown from standard image filters, such as contrast, brightness, and gamma adjustment, explained previously. Possession of the original cover image makes positive identification and attribution possible in almost all circumstances. 


\section{LOW QUALITY}

Typically, low quality steganography software simply inserts the data to be embedded into the LSB image color plane, without regard to how easily this would be possibly detected using a forensic analysis.

\subsection{Steganography Online}

http://stylesuxx.github.io/steganography/

This particular software apparently first completely zeroes out the LSB bit plane in all colors, and then encodes the data into a narrow strip at the top of the image. This is visually evident in the analysis image shown below - where all LSB bits are simply set to zero, except for the data at the top. This is the least sophisticated and most naïve of all the steganographic software we will be analyzing - the ugly.

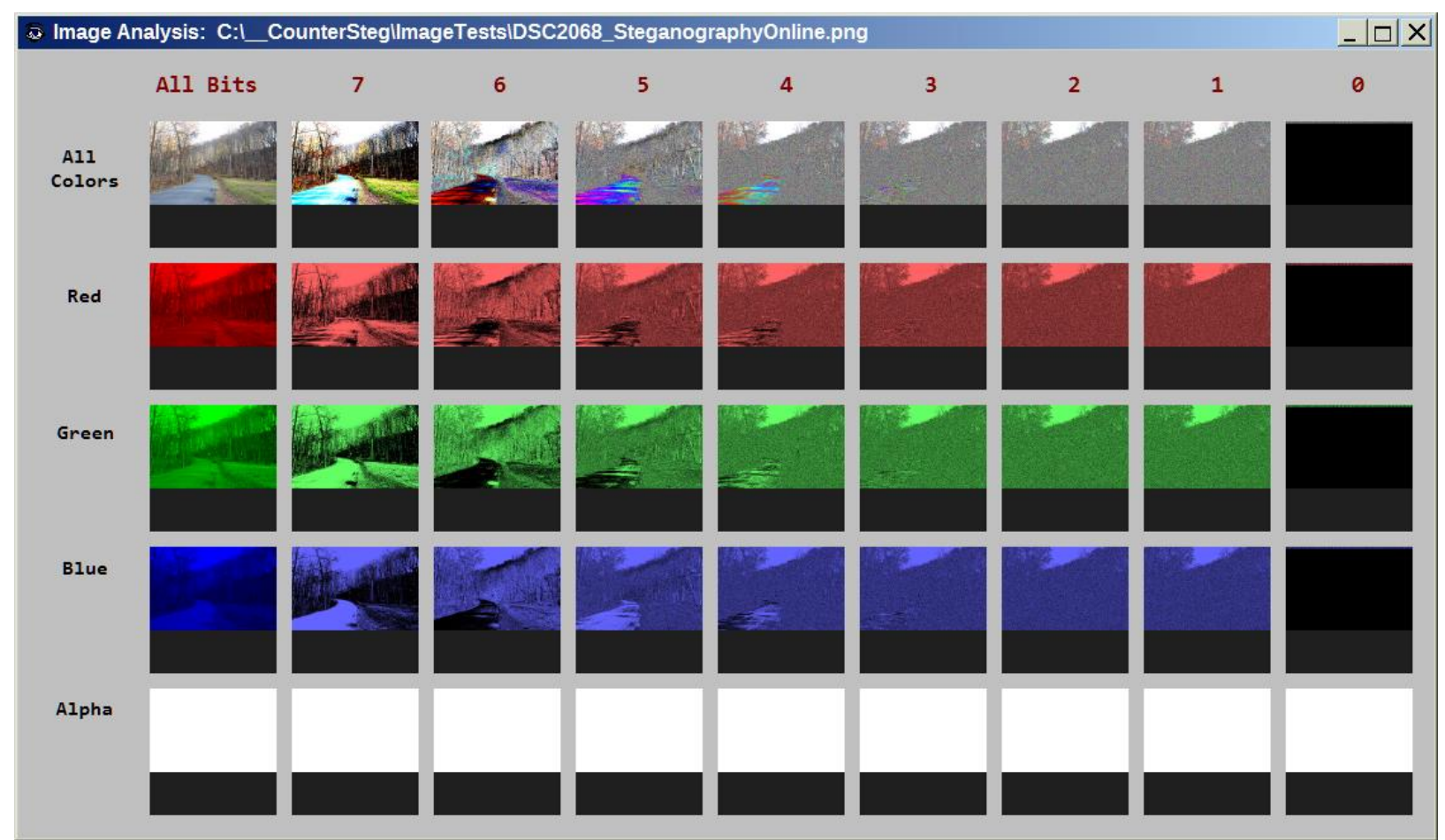

Figure 8. Analysis of image created by Steganography Online 
In the comparison image shown below, you can see all bit planes are identical from bit plane 7 to 1. All modifications take place in bit plane 0 (LSB), which is in fact first set to zero, and then the data is encoded.

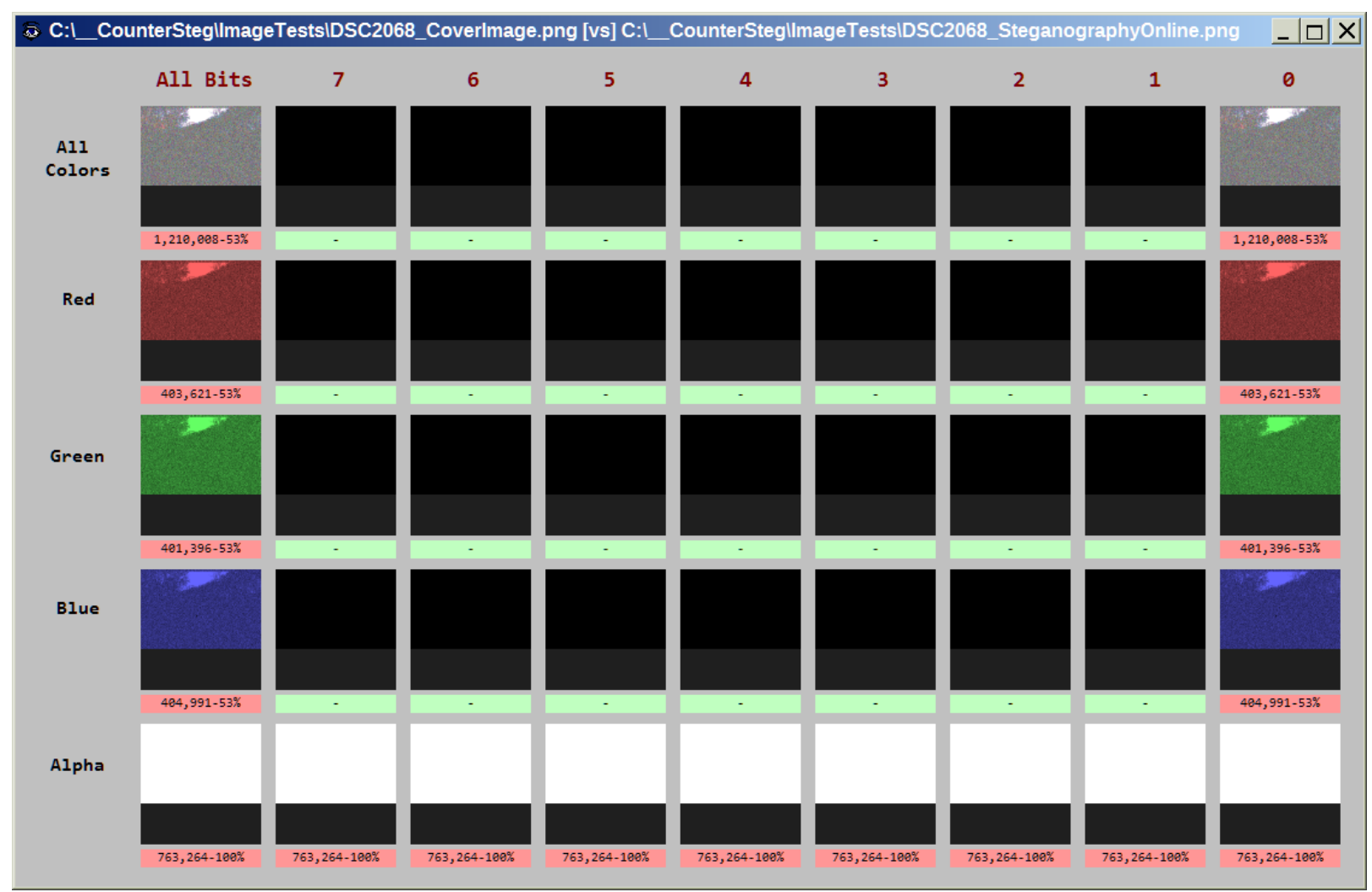

Figure 9. Comparison of cover image to image created by Steganography Online. 
The image shown below is an expansion of all colors in bit plane 0 , the data containing strip at the top is evident. In particular, the area of saturated white pixels at the top is completely overwritten. This software will be easily forensically detectable and identifiable in usage.

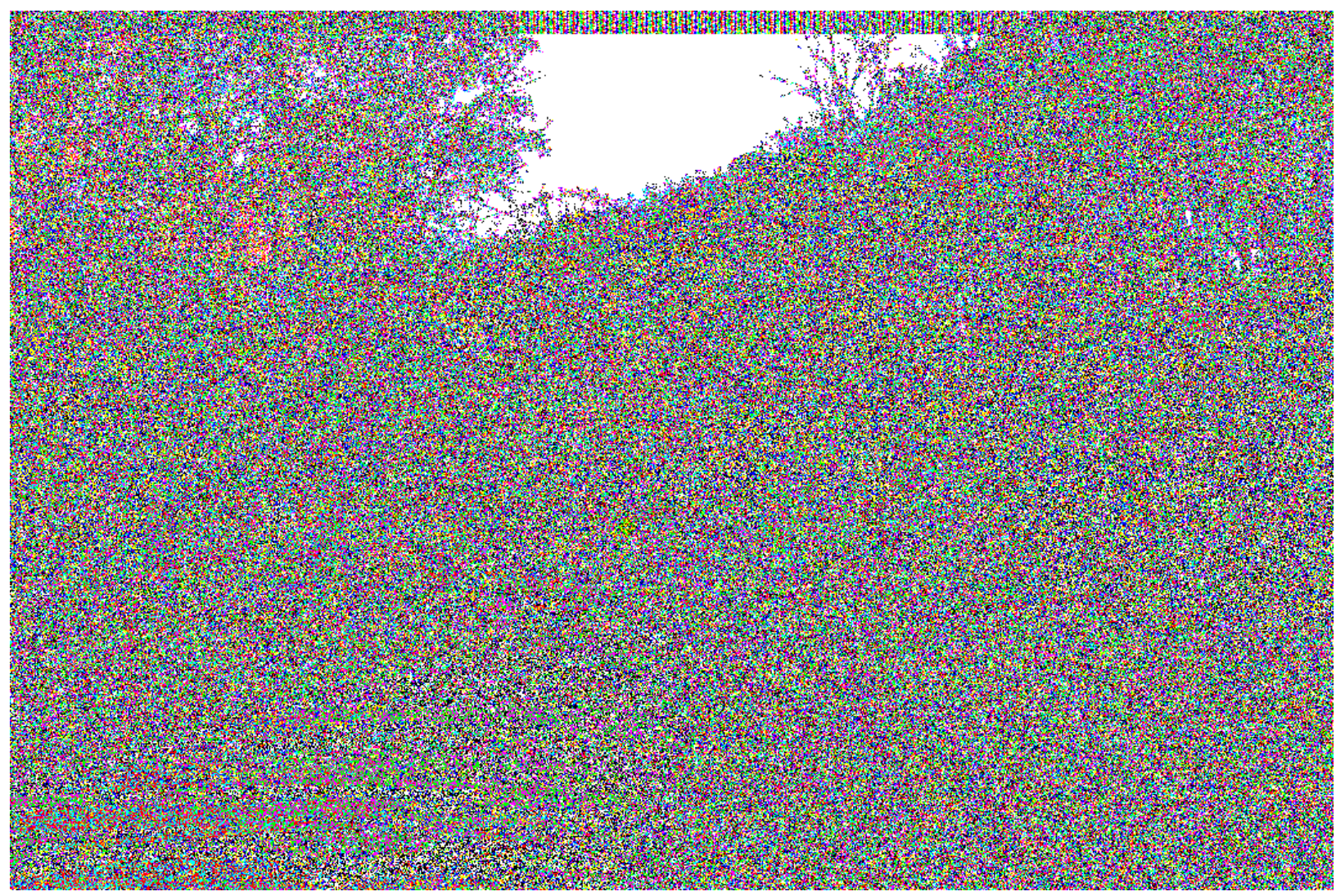

Figure 10. LSB values of Steganography Online image 


\subsection{StegoShare}

http://stegoshare.sourceforge.net/

The next poor quality steganography software uses a similar approach, however, does not completely zero out the LSB plane. In addition, it makes modifications to bit plane 1 and 2, for reasons unknown. Other bit planes remain unchanged. Further, some type of narrow strip of information is embedded in the top center of the image. Analyzing the image in bit planes 0,1 , and 2, as shown below, clearly depicts the modifications.

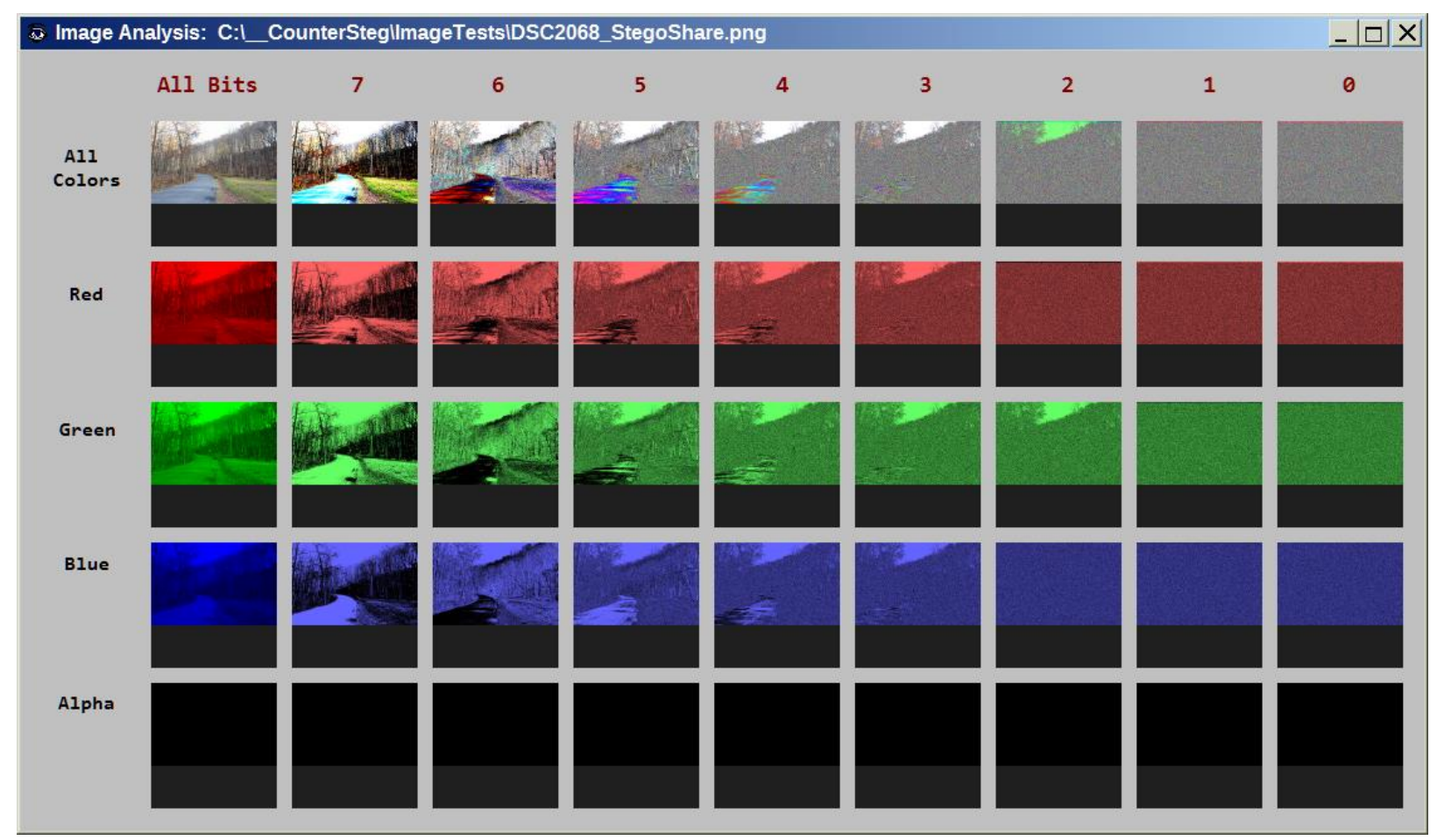

Figure 11. StegoShare image analysis. 
In comparing the images, you can see the large percentage (33-50\%) of modifications made to bit plane 0,1 , and 2, with the exception of green in bit plane 2 .

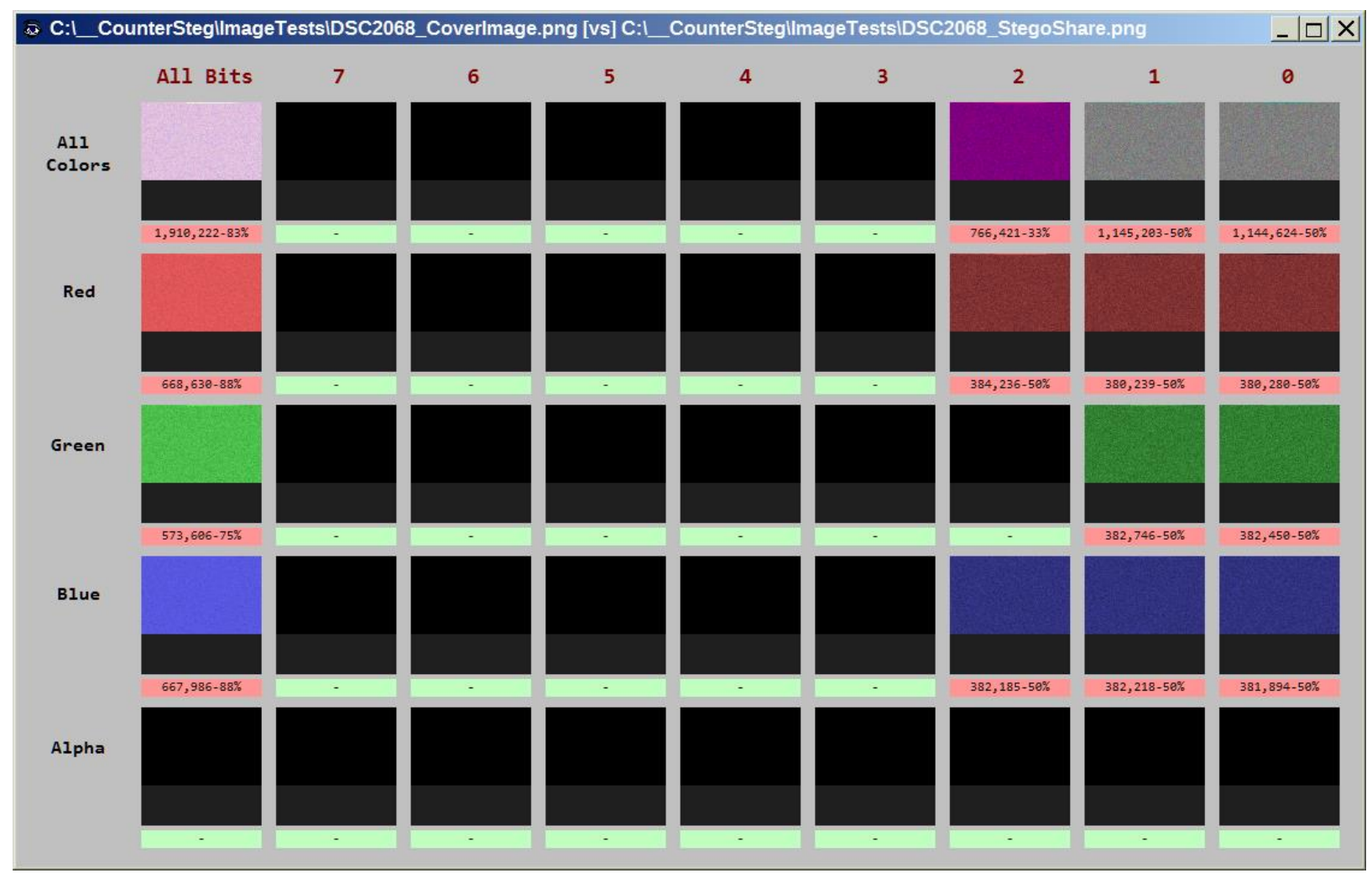

Figure 12. StegoShare image comparison. 
The expansion of the changes to all colors in all bit planes image shown below shows the narrow strip of information also embedded into the top center of the image. This is shown in Figure 13 that follows.

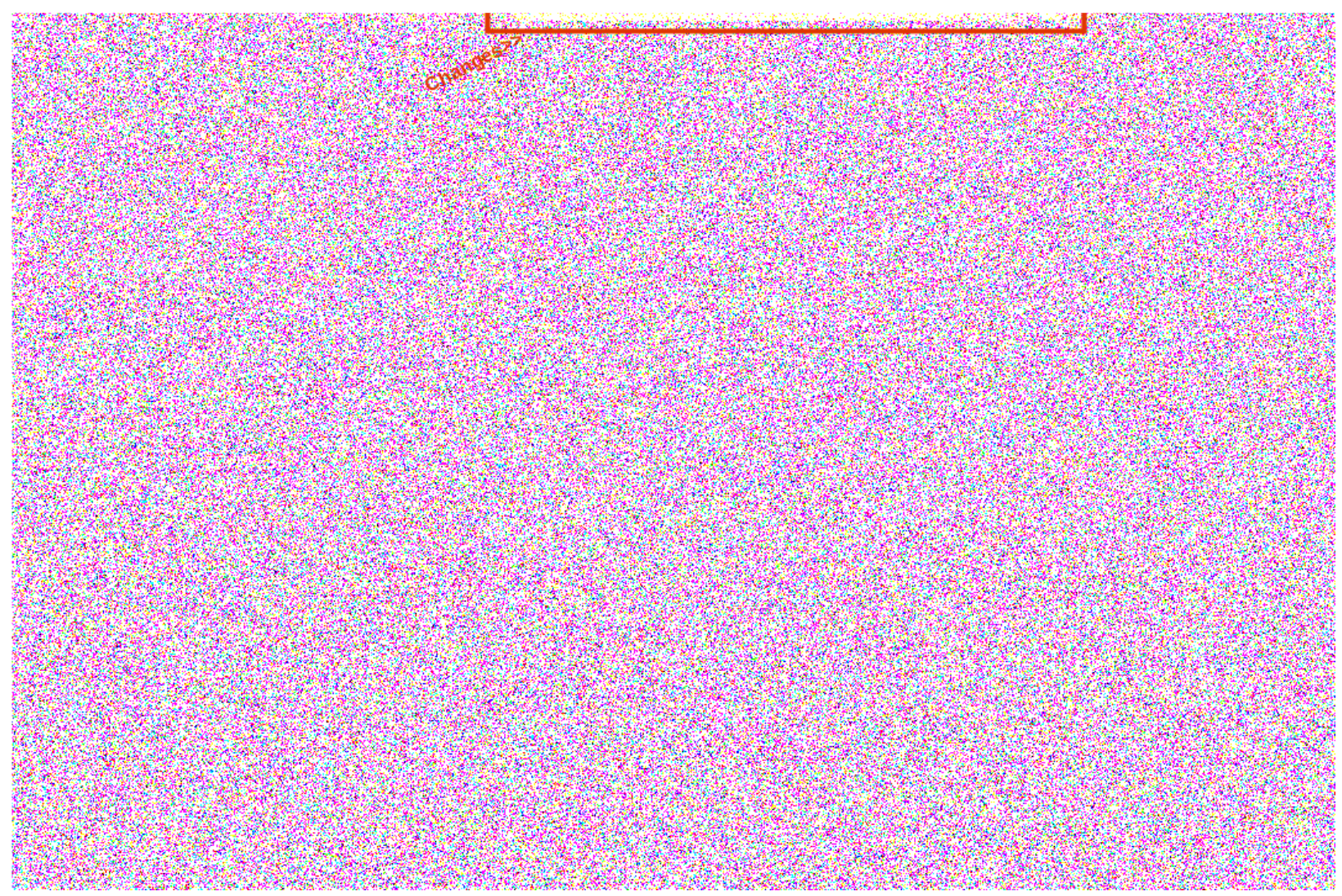

Figure 13. StegoShare LSB values. 


\subsection{Geocaching Toolbox}

https://www.geocachingtoolbox.com/index.php?page=steganography

This software only alters the LSB bit plane in all three colors, however it includes all the data into a narrow strip at the bottom of the image. This would be easily detectable using RS statistical analysis as changes to the bit patterns in only a small fraction of the image (the portion containing the data). The overall analysis of the image, as shown below in Figure 14, does not indicate much forensically. In this case we also need the original cover image for comparison, which then makes the positive conclusion evident.

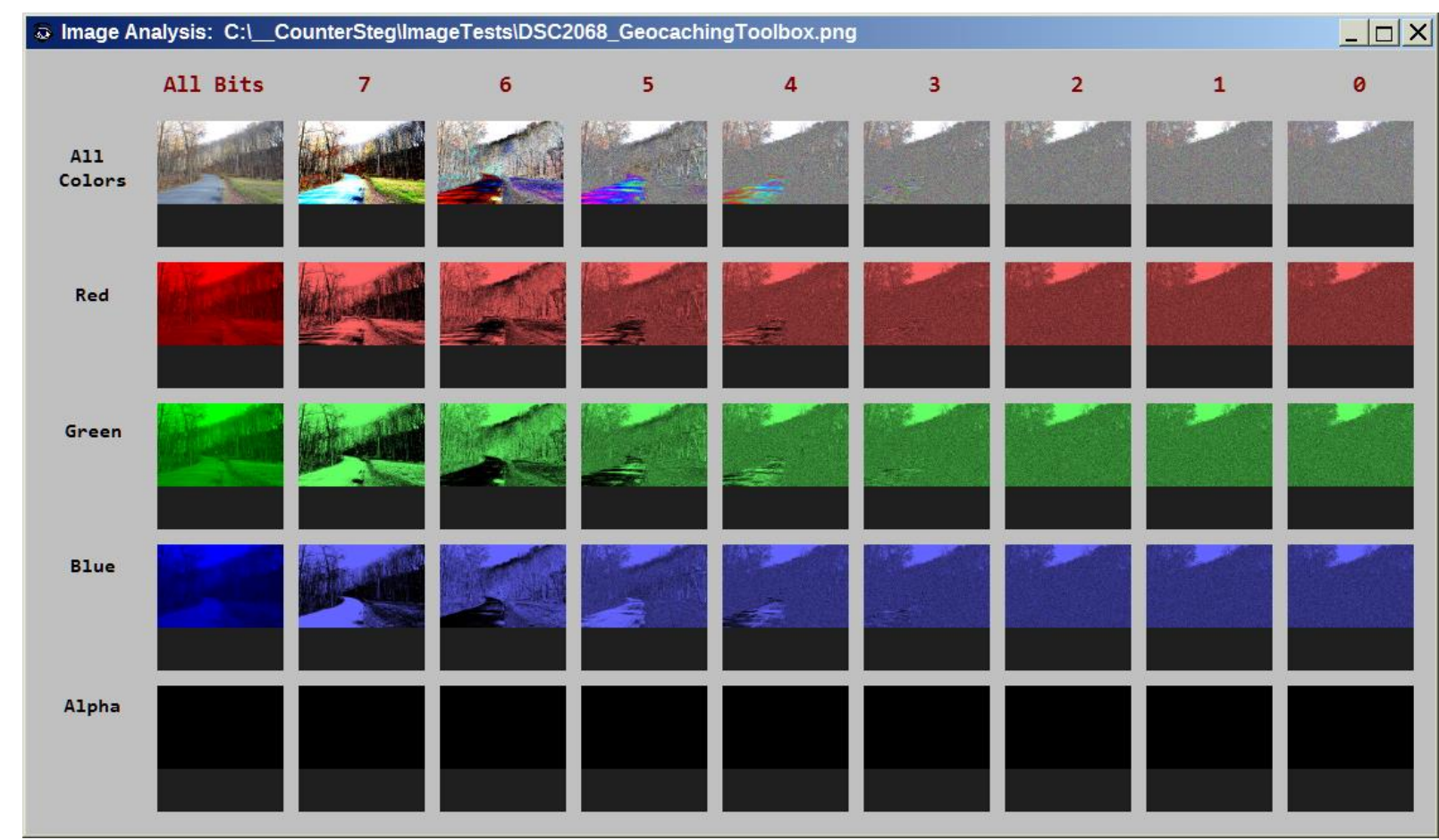

Figure 14. Geocaching Toolbox image analysis. 
Below in Figure 15 is the comparison image - showing the data payload embedded to the narrow strip in the bottom of the image. However, due to the small amount of pixel changes (1\%), it is likely at least the software compresses the data before embedding. Compressing the text data before embedding typically can reduce the size of the necessary modifications to the image by 80 to $90 \%$. As a result, higher quality steganographic software will always compress data before engaging in the image embedding process.

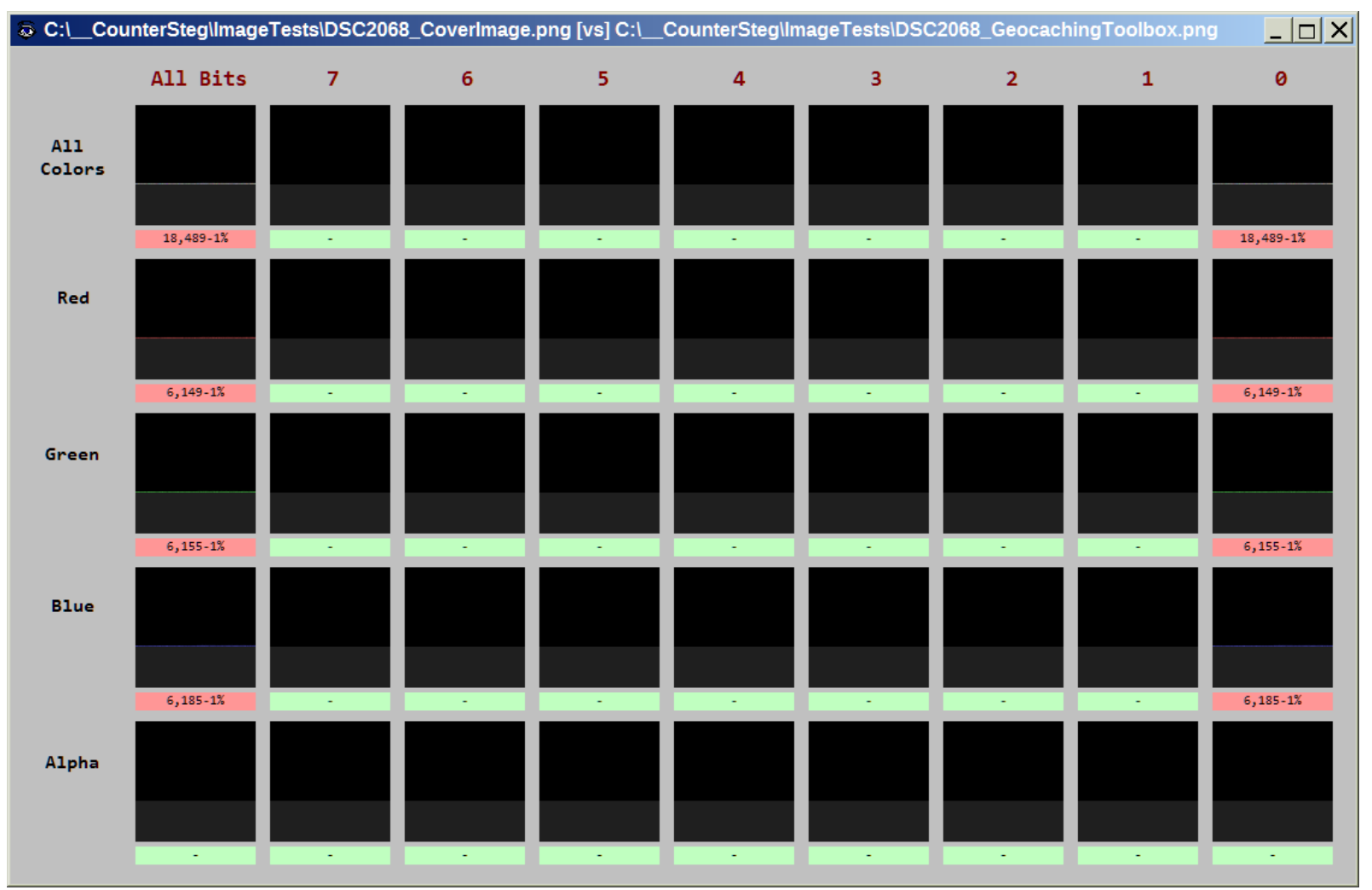

Figure 15. Geocaching Toolbox image comparison. 
The narrow strip is clearly visible in Figure 16 at the bottom.

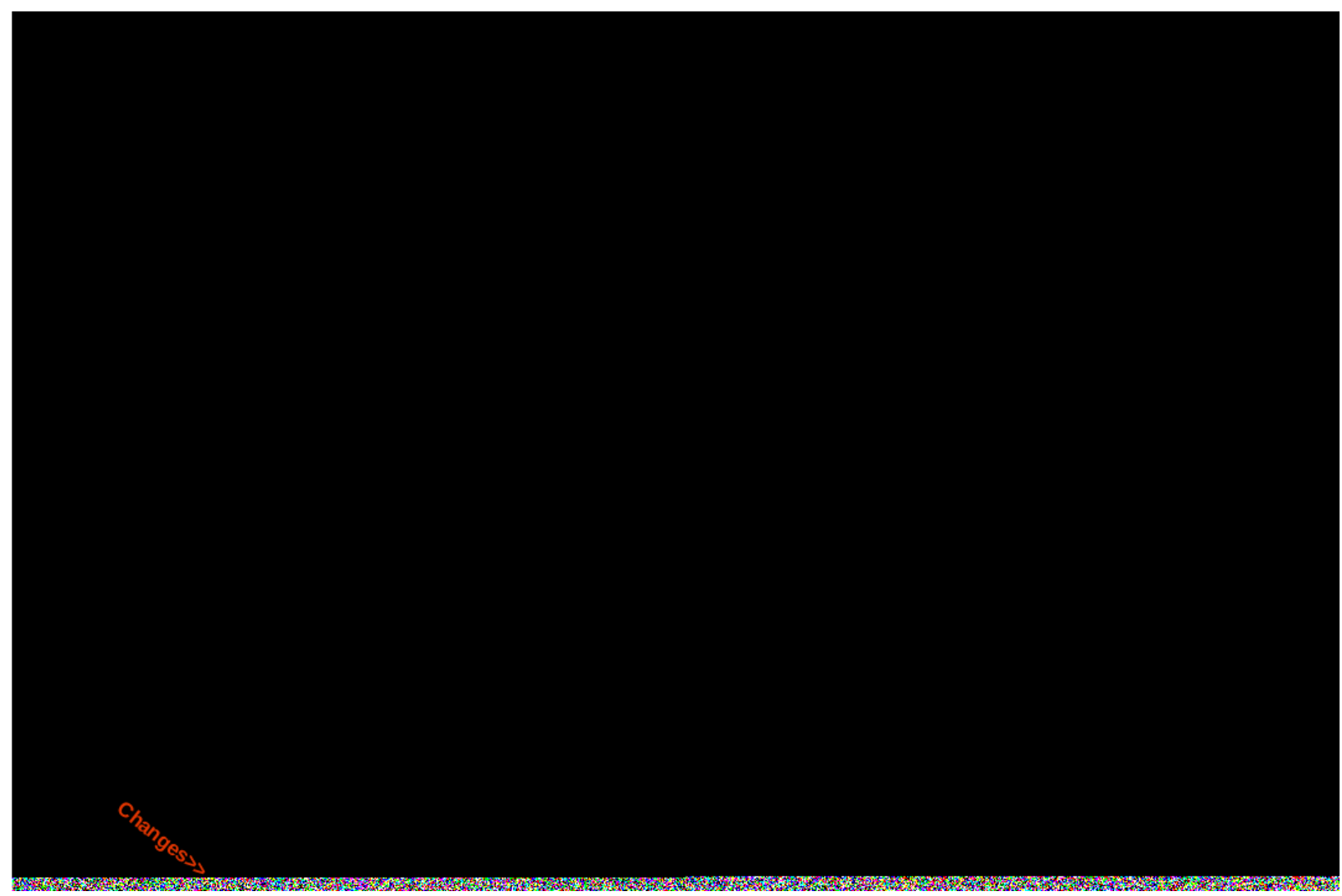

Figure 16. Geocaching Toolbox LSB changes. 


\subsection{DevFarmSteganography}

https://devfarm.it/steganography/

This software is comparable to the previous software, Geocaching Toolbox, and may make use of the same steganographic embedding libraries. The modifications to the image are very similar, with only slight differences in the number of pixels modified.

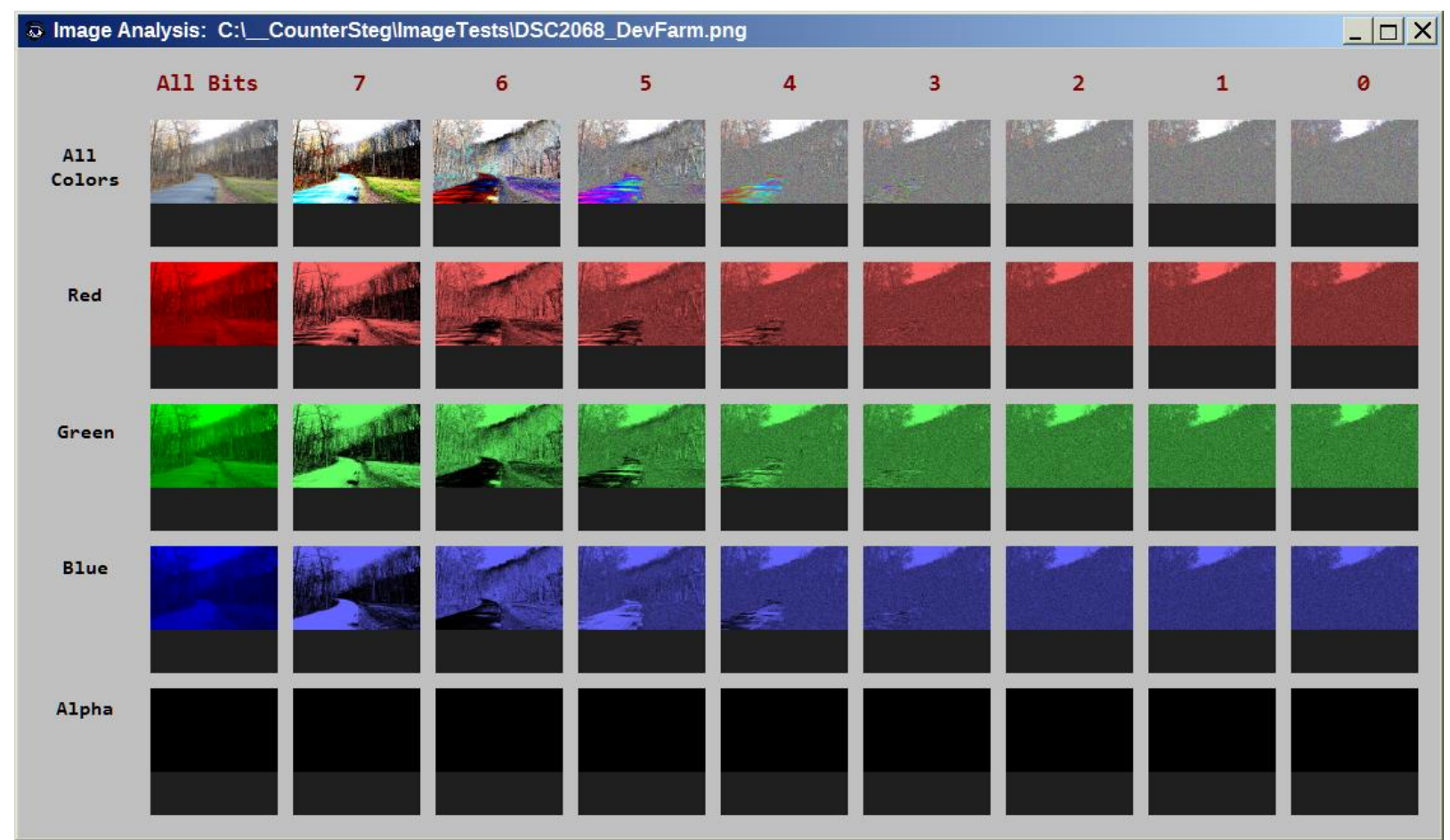

Figure 17. DevFarm Steganography image analysis. 


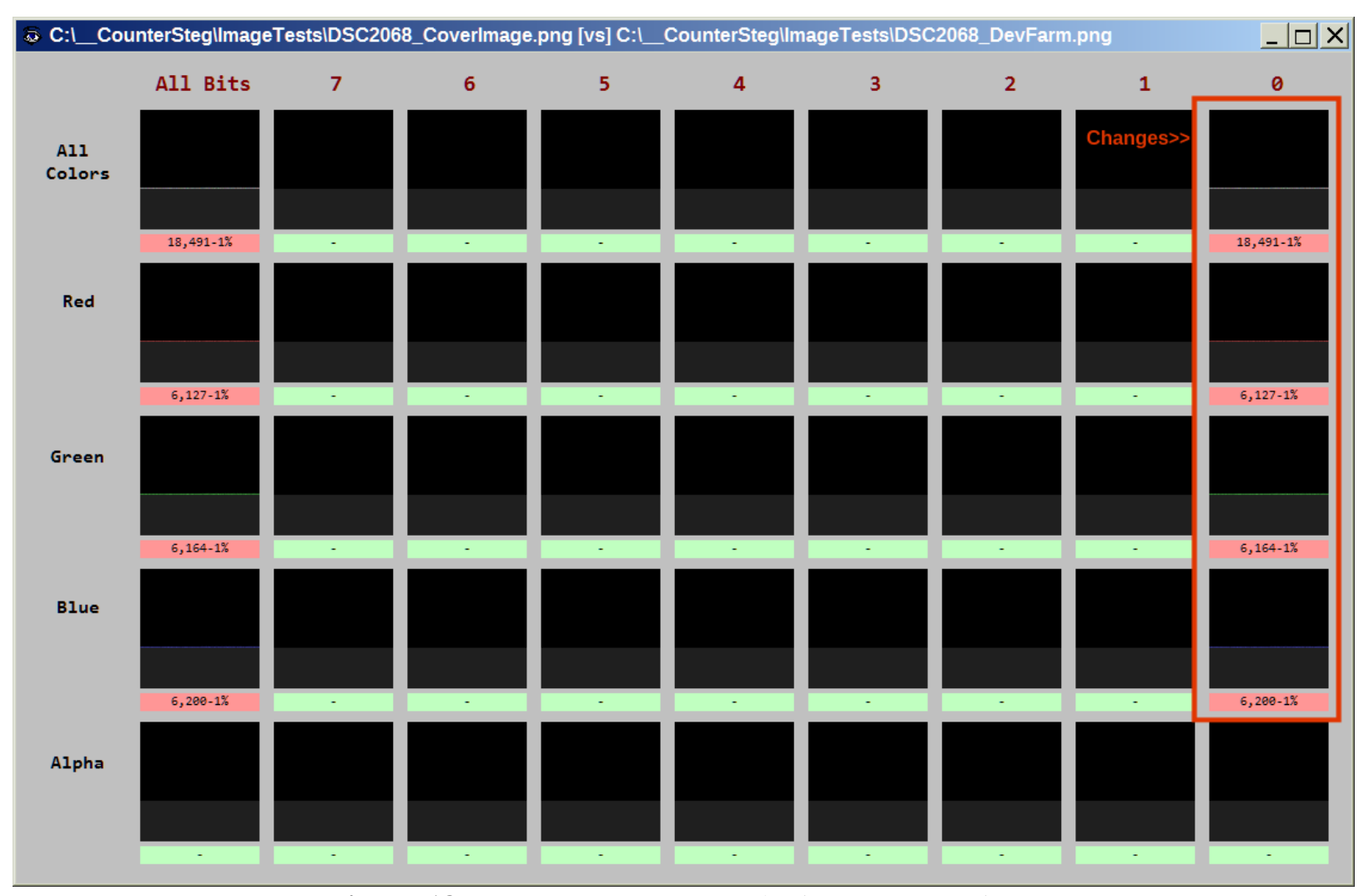

Figure 18. DevFarm Steganography image comparison. 
Figure 19. DevFarm Steganography LSB image changes. 


\section{MID-RANGE QUALITY SOFTWARE}

The mid-range quality software is an improvement over the ugly, at least narrow strips of pixels are not apparently encoded (and more easily detected), however security shortcomings are still evident.

\section{1 f5stego.js}

http://desudesutalk.github.io/f5stegojs/

This software seems to take the unique approach of ignoring the LSB and simply encoding data depending whether color values are odd or even. Even though many of the pixel colors are modified through multiple bit planes, some of the color values remain unchanged. This is evident in the comparison image of all bits in all colors shown as Figure 21.

An analysis of the image shown in Figure 20 does not show undue pixel modifications or strips. However, due to the large amount of color changes, visual differences will be evident between the original and modified image.

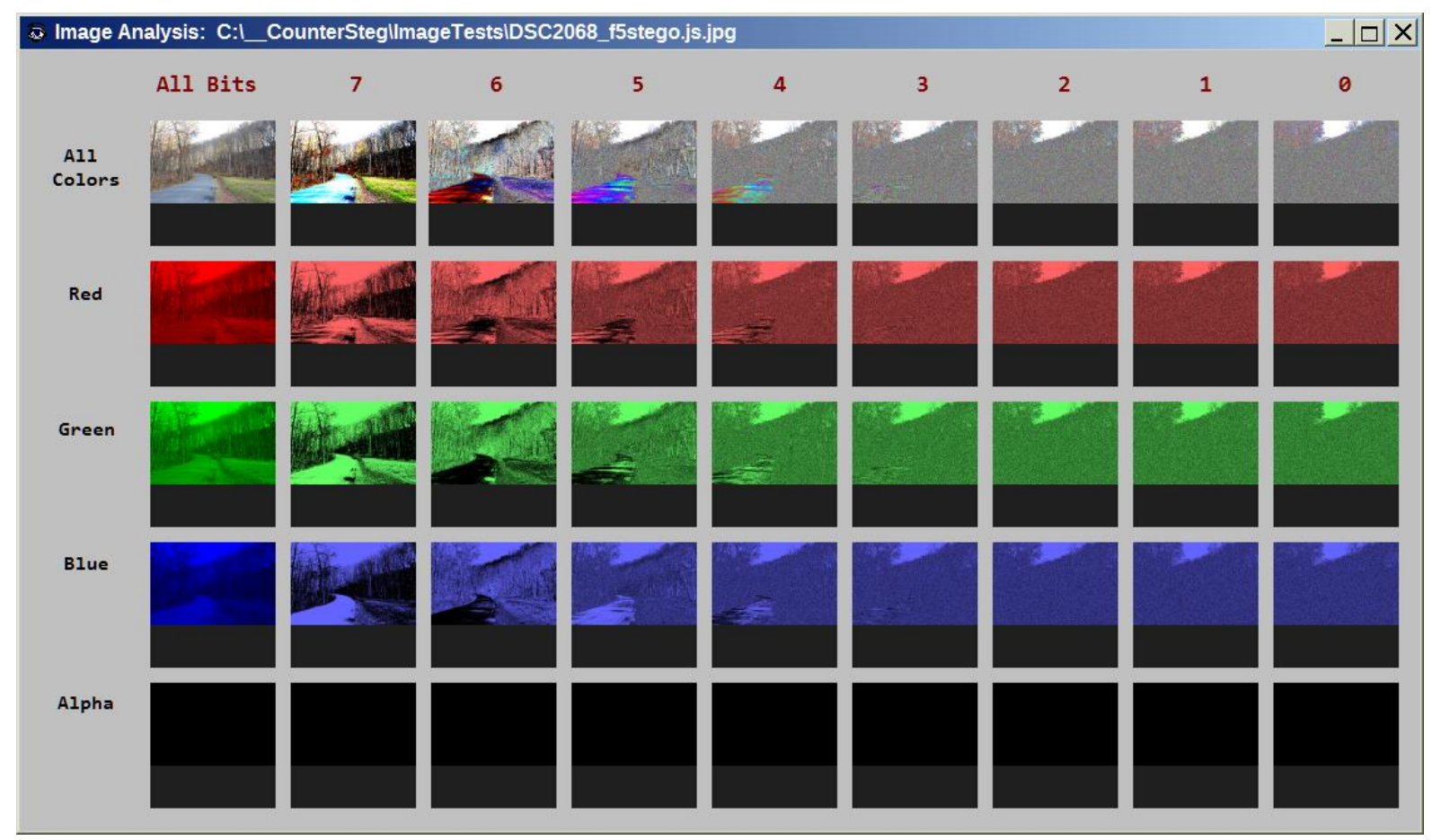

Figure 20. f5stegojs image analysis. 
Below in Figure 21 is the comparison analysis. Notice that all bit planes and all colors are modified. On first analysis, this would appear to be very similar to the contrast adjustment shown previously, however in that case virtually all pixels are modified, except for the saturated white area. In this case, many pixels remain unchanged - hinting at the possibility of "all bit" encoding. In other words, data is encoded by overall color intensity value for the respective color channel, red, blue, or green (in the range 0 to 255). Depending on whether the color intensity is odd or even, this indicates the value of the bit for that particular color channel. Three bits can be encoded for each pixel in this fashion. However, forensic analysis of the steganographic image easily identifies a payload because of the fact that only selective pixels are modified.

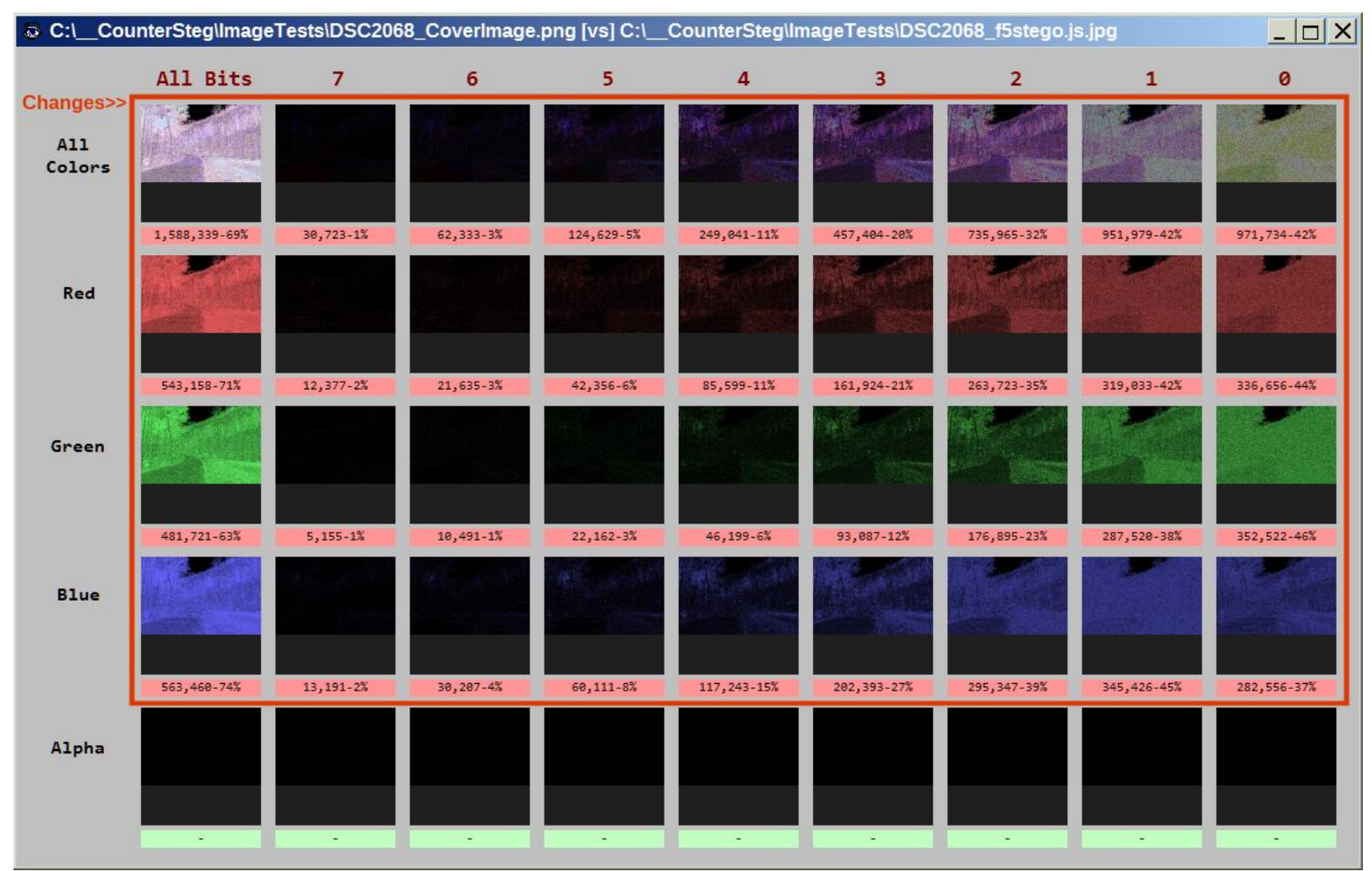

Figure 21. f5stegojs image changes. 
As shown below in Figure 22 in the expansion of the comparison image in all color LSB bits - many pixels remain unchanged in an apparent random fashion. It is likely saturated pixels are avoided (an aspect of quality in this software), and random noise is added in areas of the image not needed for further data encoding. Overall, $69 \%$ of the pixel color values in this image have been modified.

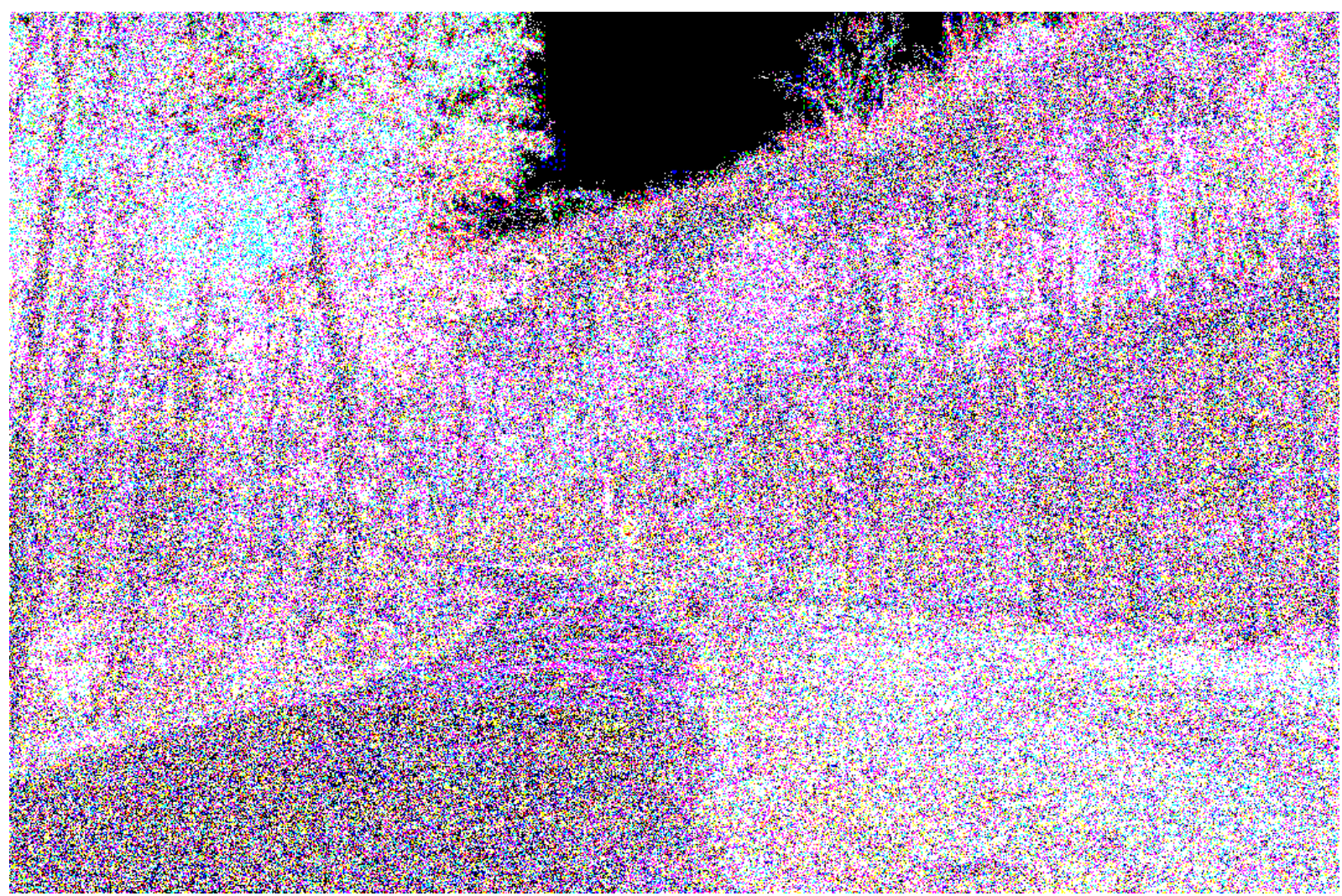

Figure 22. f5stegojs LSB image changes. 


\subsection{BitCrypt}

http://bitcrypt.moshe-szweizer.com/

BitCrypt fails in the area of modifying saturated white pixels, and makes this software more easily detectable. The sophisticated forensic analyst will be aware that camera CMOS sensors typically saturate to maximum values in bright areas, such as the sky, will not vary between colors pixel to pixel. These block areas will be fully saturated and will remain so through the area of the similar object, such as the overcast sky. Modifications to pixels in these areas will be telltale signs for software image message or data carrying modifications.

An overall analysis of the image shows the bit 0 and 1 modifications in the area of saturation. Bits 2-7 appear to be largely unchanged in the area of saturation, as shown below in Figure 23.

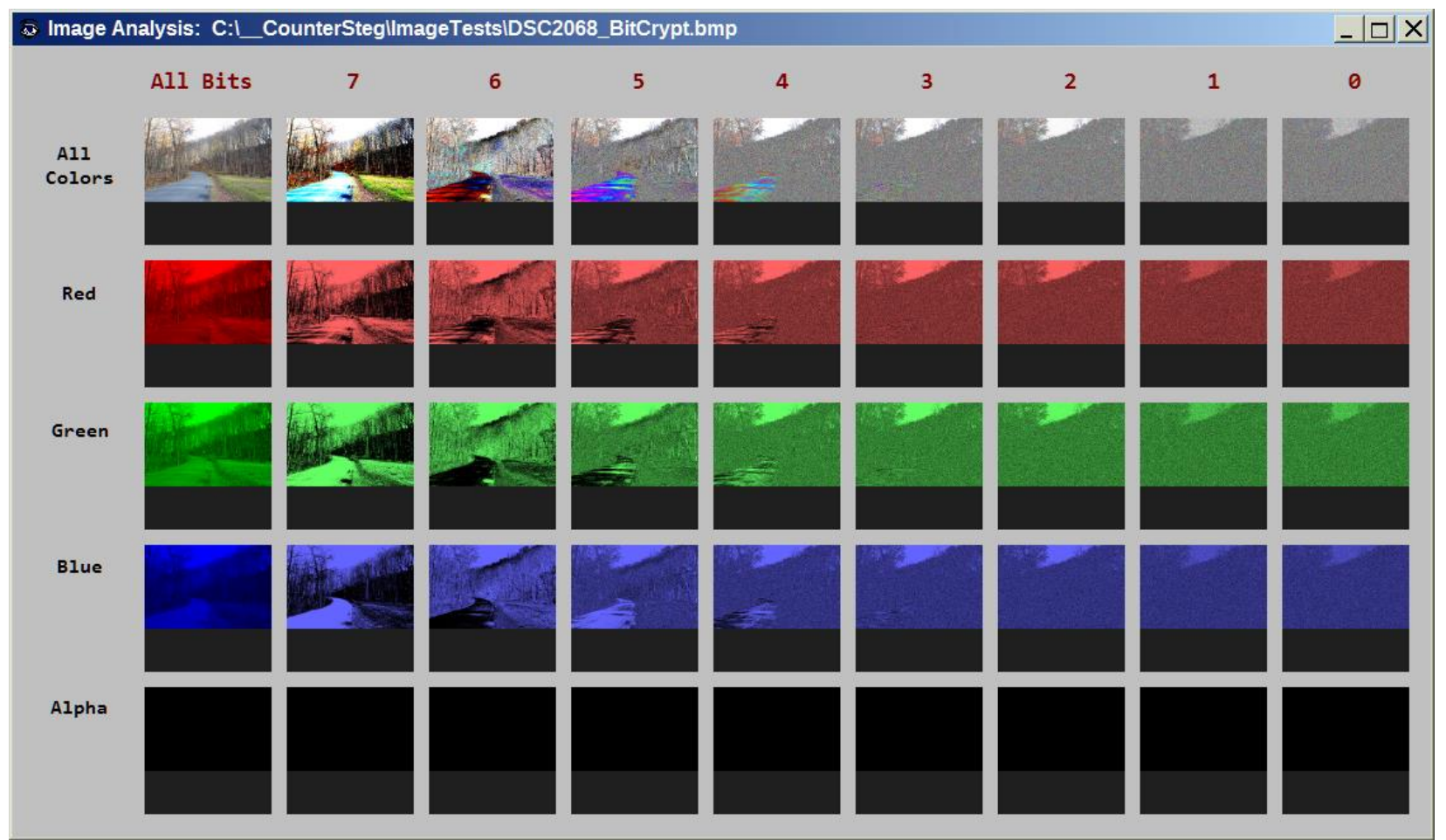

Figure 23. BitCrypt image analysis. 
Comparison with the cover image shows the broad modification of pixel colors in bits 0 through 7, however the area of saturation is avoided above bit 1 . This most likely is a software coding implementation to create a similarity with a standard image processing function, such as brightness, contrast, or gamma adjustment. However, in those cases the changes in the saturated area will propagate up through bit 7 , and including bit 7.

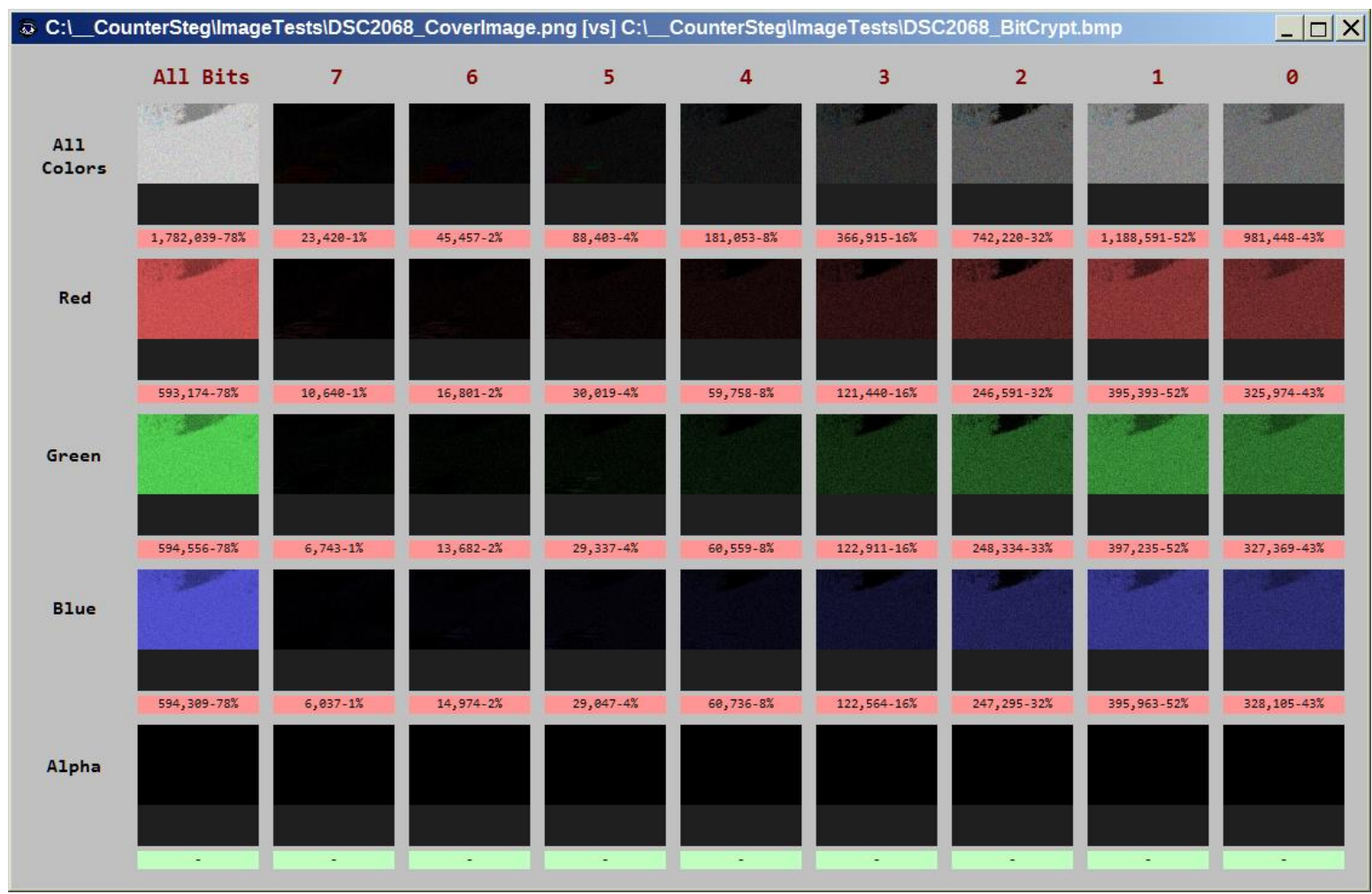

Figure 24. BitCrypt image comparison. 
Further, the expansion of the bit 0 in all colors graphic analysis depicts the seemingly random dispersal of LSB bit changes, except for the relative lack of changes in the saturated area. This particular forensic pattern for steganographic activity is indeed unique. While subtly different from standard image processing comparison results, the bit modification pattern should still be able to be classified and identifiable. Further test images should be created and comparisons conducted in the future to analyze and identify with further clarity the modifications BitCrypt makes to images.

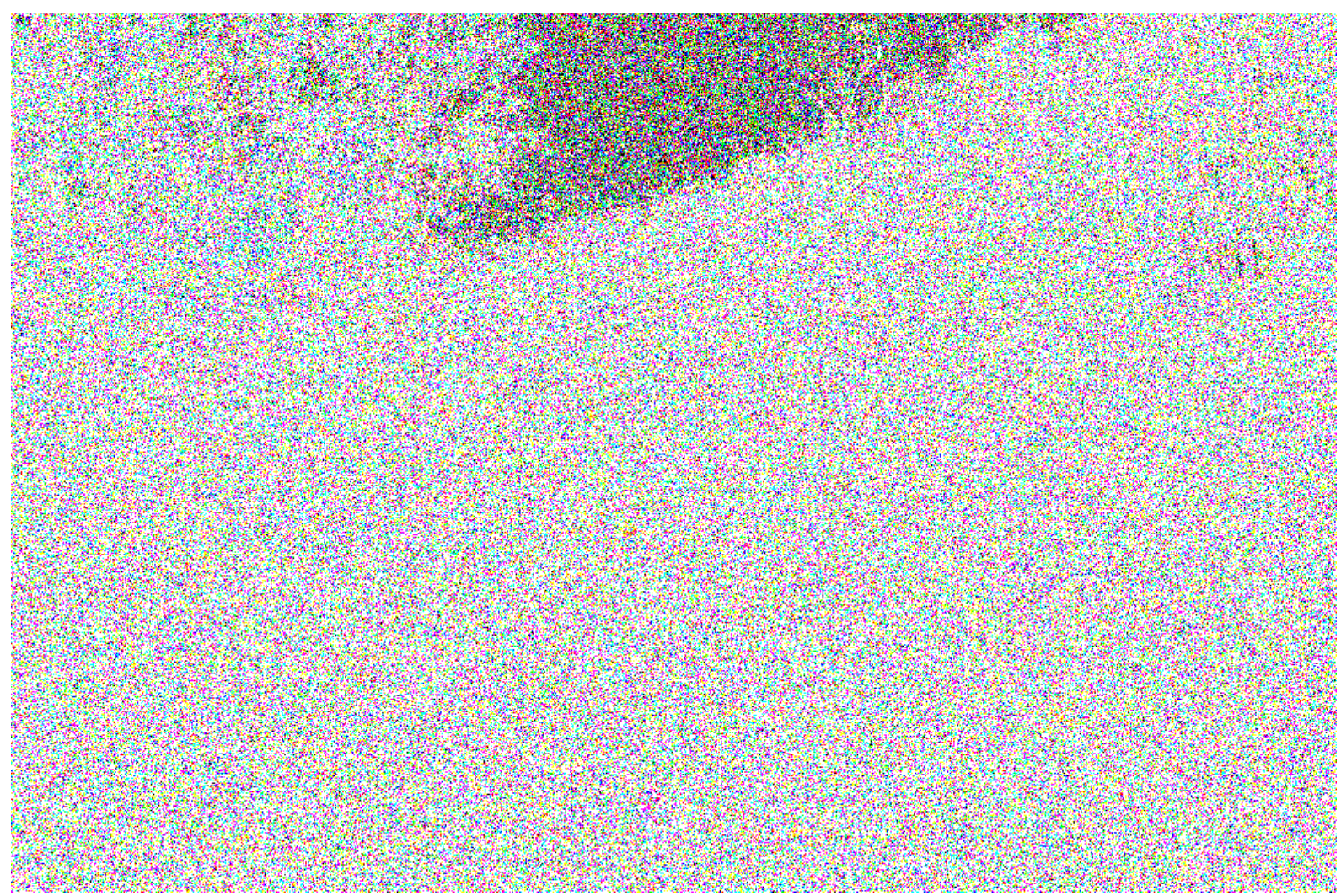

Figure 25. BitCrypt LSB image changes. 


\subsection{OpenPuff}

http://embeddedsw.net/OpenPuff_Steganography_Home.html

While completely avoiding the saturated pixel area, which is good, OpenPuff attempts to modify far too many pixels to not be detectable especially with a comparison image in hand. The software only modifies pixels in the LSB plane, making it virtually undetectable visually. However, overall $12 \%$ of the pixels are modified in the image - compare this to $1 \%$ even with less sophisticated programs. Statistical analysis of the image will reveal changes to typical photograph LSB bit patterns in typical similar photos with similar CMOS sensors.

Overall analysis of the image, as shown below in Figure 26, does not reveal any particular fine points for analysis - making OpenPuff the best of the bad software for Steganography in this paper analysis.

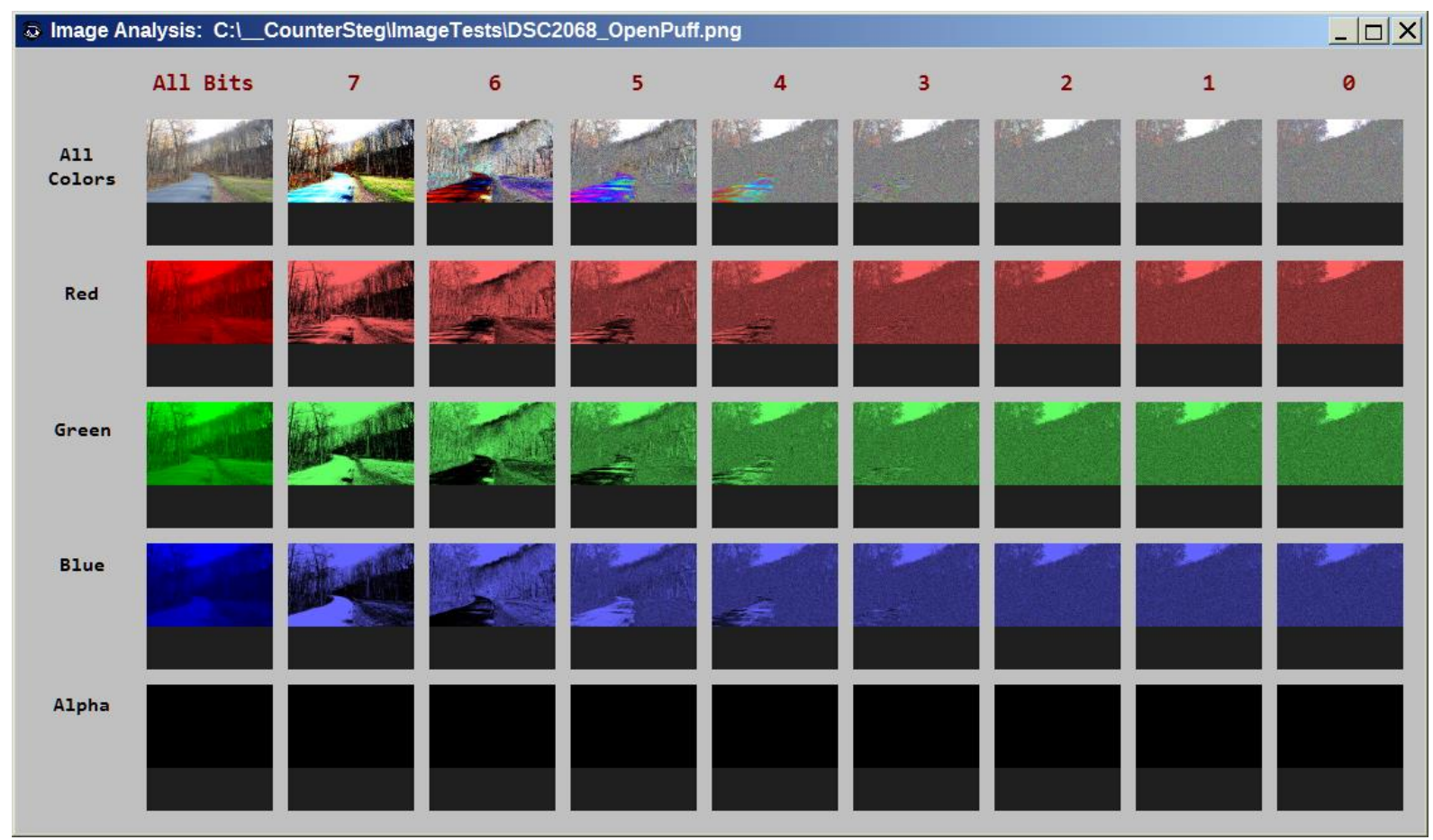

Figure 26. OpenPuff image analysis. 
As shown below in Figure 27, OpenPuff only makes changes to the LSB values in the image but does these too far too great an extent at $12 \%$. This leaves the payload image vulnerable to statistical analysis techniques. With a comparison image in hand, since only LSB values are modified, it is highly probable to conclude steganographic payload has been embedded into the image.

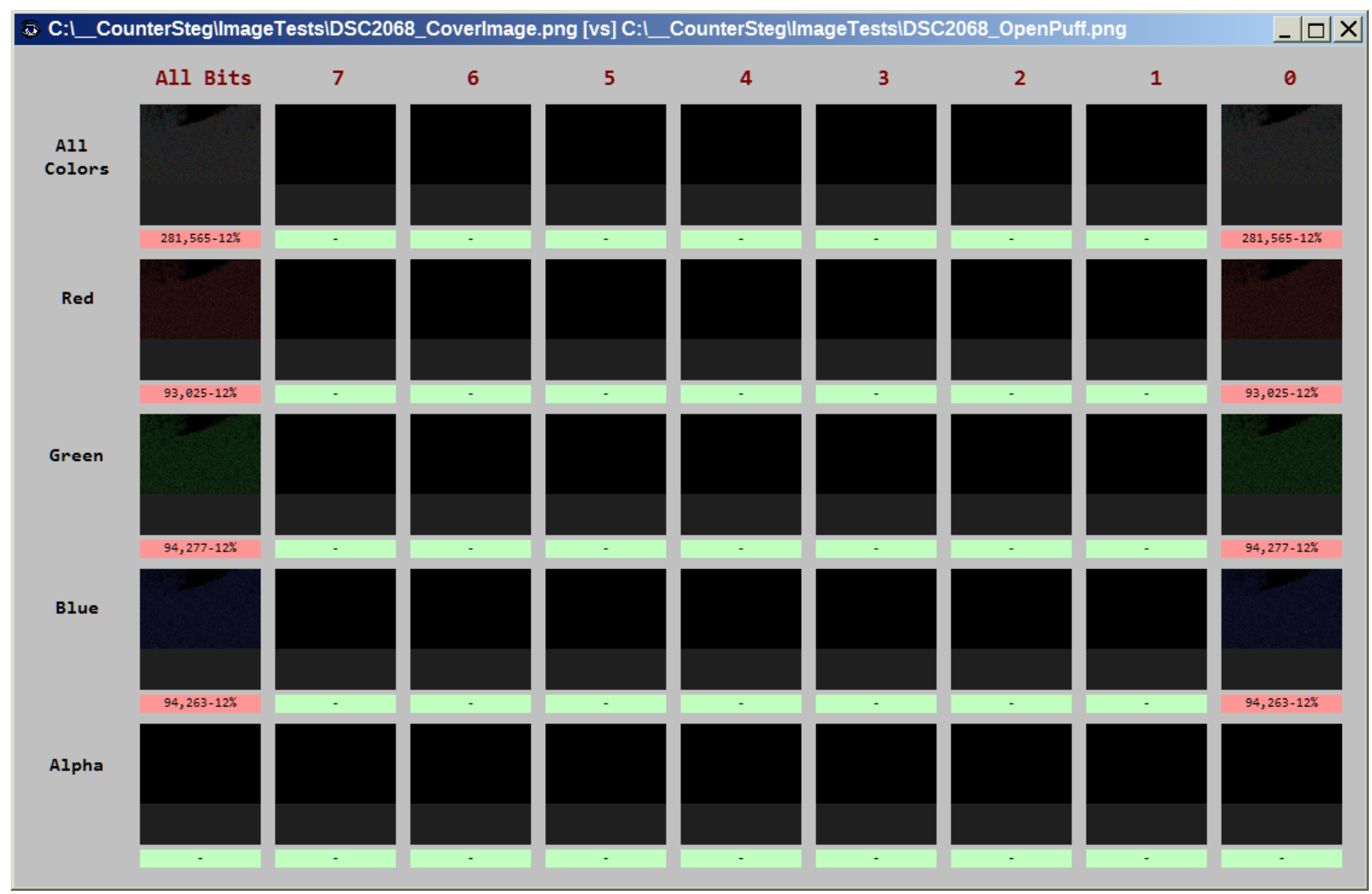

Figure 27. OpenPuff image comparison. 
Further, as shown below in Figure 28, the software takes care to avoid saturated color pixel areas. This is commendable. However, without exception, all other areas of the image are highly modified — possibly with random data. This apparently random data will be more apparent to sophisticated image forensic analysis techniques.

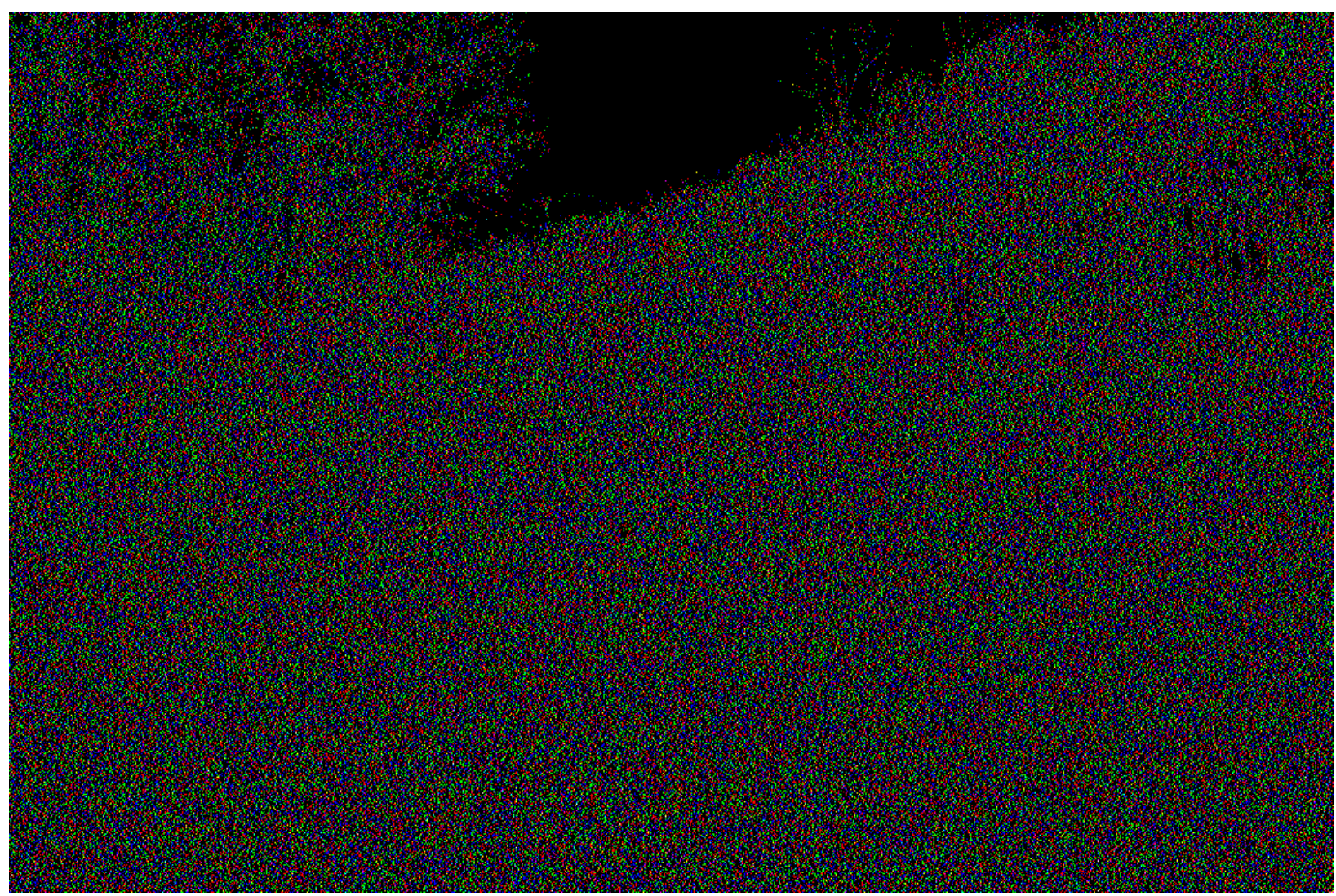

Figure 28. OpenPuff LSB image changes. 


\section{HIGH QUALITY}

The high quality steganographic software available in general attempts to do several things. It creates a low payload profile - in our examples below $1 \%$ of the pixel values only of the image are modified. This compares to the $12 \%$ to $83 \%$ of the pixels modified by the other software analyzed previously. Less modifications will correspond to and equal less detectability. Also, it is important to disperse the changes into various areas of the image, specifically areas of higher noise and color variation. Areas of solid colors or pixel saturation should be actively and strongly avoided to lower the possibility and probability of forensic detectability.

\subsection{OpenStego}

https://www.openstego.com/index.html

OpenStego takes care to compress the data before embedding, reducing the overall payload size to about $1 \%$ of the image pixels. Also, it disperses the data encoding seemingly randomly throughout the image.

The overall image analysis shown below in Figure 29 provides little for further examination if only the payload image exists. It is likely any known statistical analysis technique will fall flat and fail when trying to determine if any data modifications have been performed on the existing image in hand.

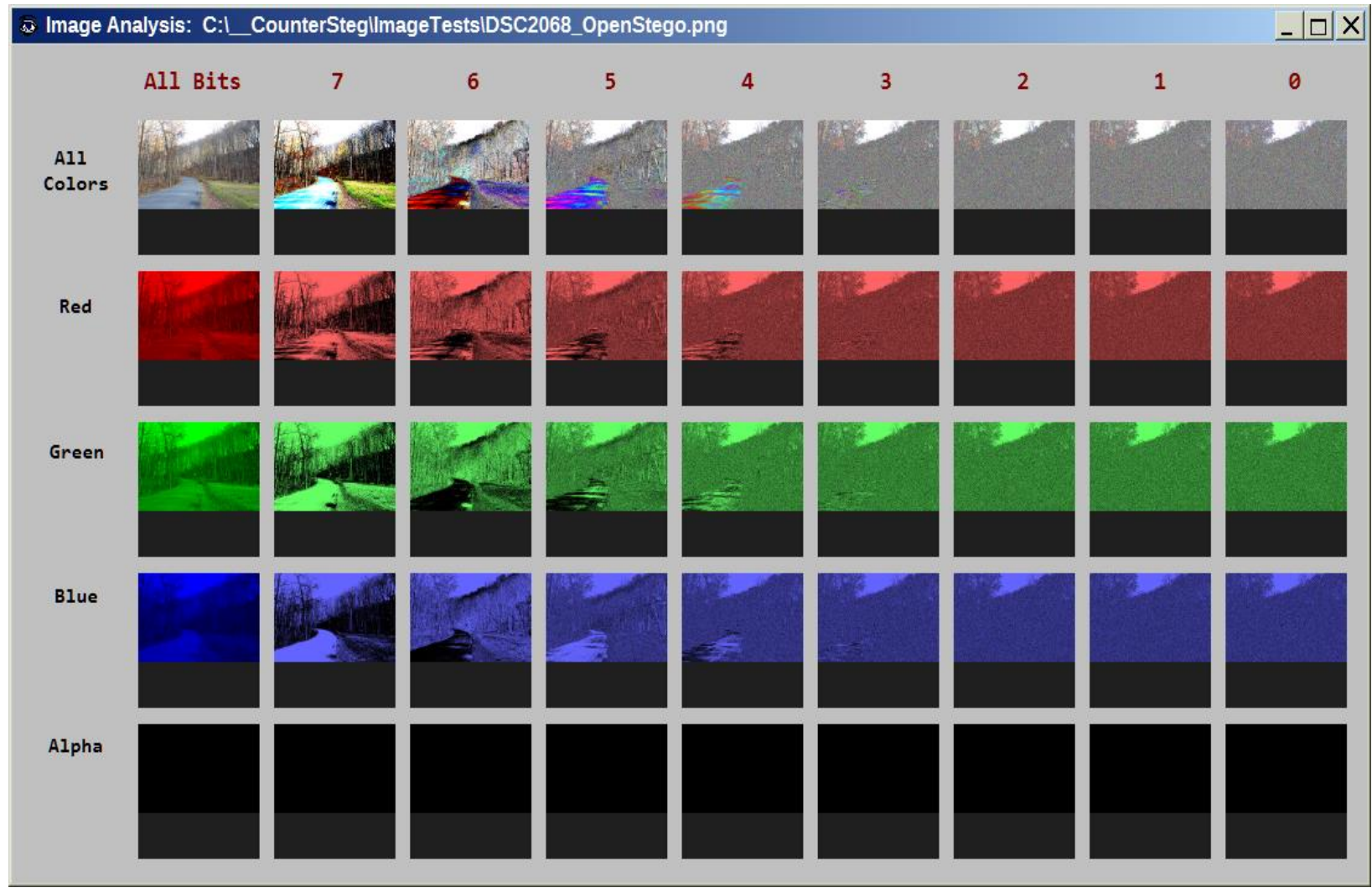

Figure 29. OpenStego image analysis. 
The comparison analysis window shown below in Figure 30 indicates only LSB values have been modified, in all three-color channels. The color channels share equally with modifications, at about $1 \%$ each. OpenStego, therefore, is not taking into account relative individual color variations when deciding where to embed data values in various color channels. No modifications are made to the alpha (transparency) color channel.

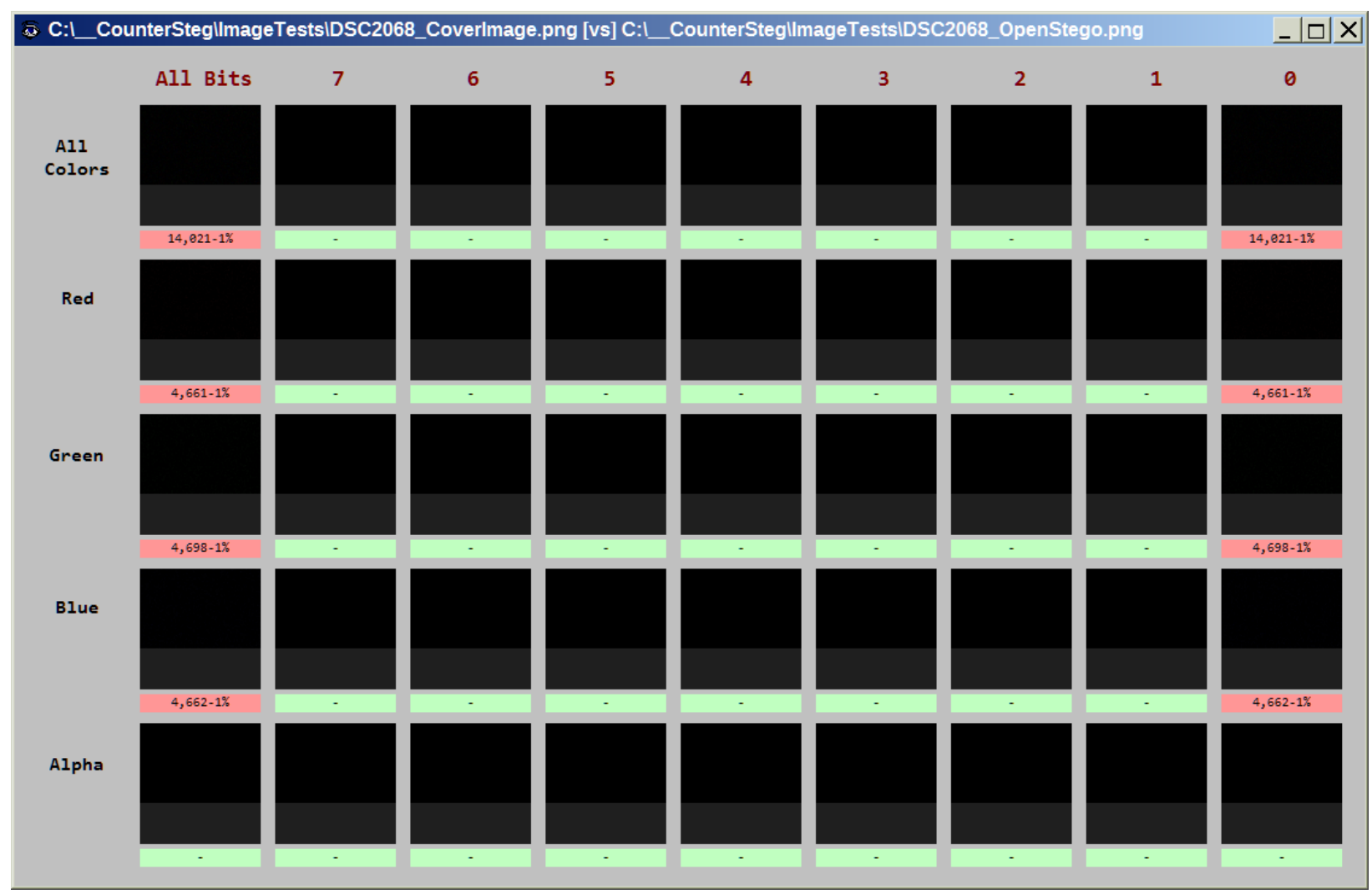

Figure 30. OpenStego image comparison. 
Below in Figure 31 are shown the LSB modifications in each color channel. Apparently, this is a random distribution not taking into account color variations throughout the image as previously mentioned. Also, the software does not apparently take into consideration noise levels or saturation levels as well when encoding data.

Figure 31. OpenStego LSB image changes. 


\subsection{OTP-Steg}

http://www.mauisolarsoftware.com/OTP-Steg/

OTP-Steg receives its name from one-time pad encryption, which is used for encrypting the data in this software before compression and encoding of the data. OTP-Steg uses the zlib library to compress all data heavily before encoding. Further, the encoding process looks for, and avoids, saturated color or solid color areas of the image. It conducts a noise and variation color analysis of the entire image, to prioritize encoding of LSB data into less statistically detectable areas.

Overall payload image analysis shown below in Figure 32 shows no features different from a standard photograph in all bit planes. Also, the payload image will be visually indistinguishable from the cover image, as only LSB values are modified.

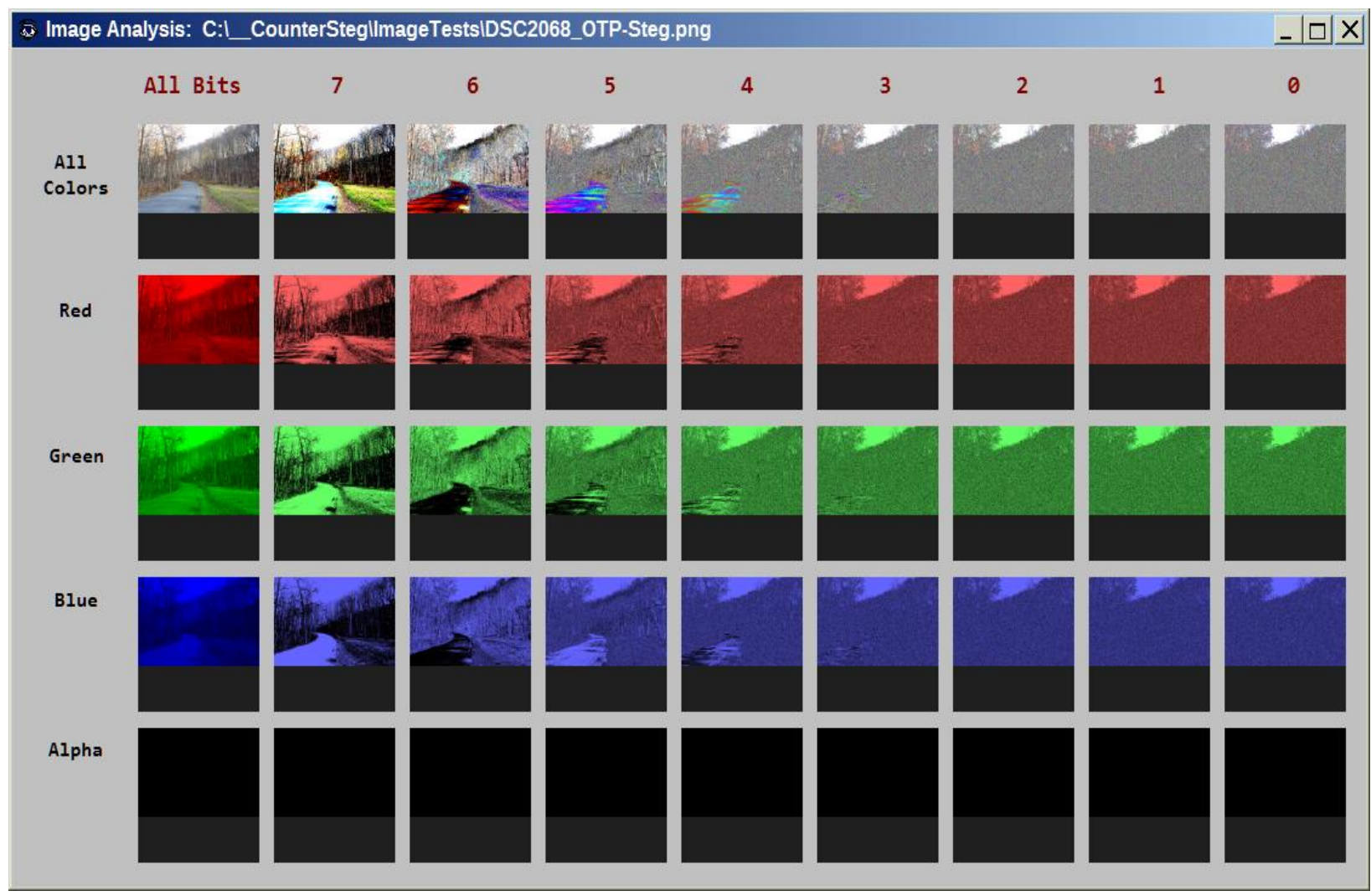

Figure 32. OTP-Steg image analysis. 
The comparison to the cover image window shown below in Figure 33 indicates only $1 \%$ of the LSB values have been modified. Notice this also varies significantly between color channel, with the red color channel carrying more than double the respective payloads of the green and blue channels. This is because red color variation varies much more significantly through the image than the green and blue color variations (Chan and Cheng, 2004). Thus, data carried in the red channel will be much harder to detect statistically.

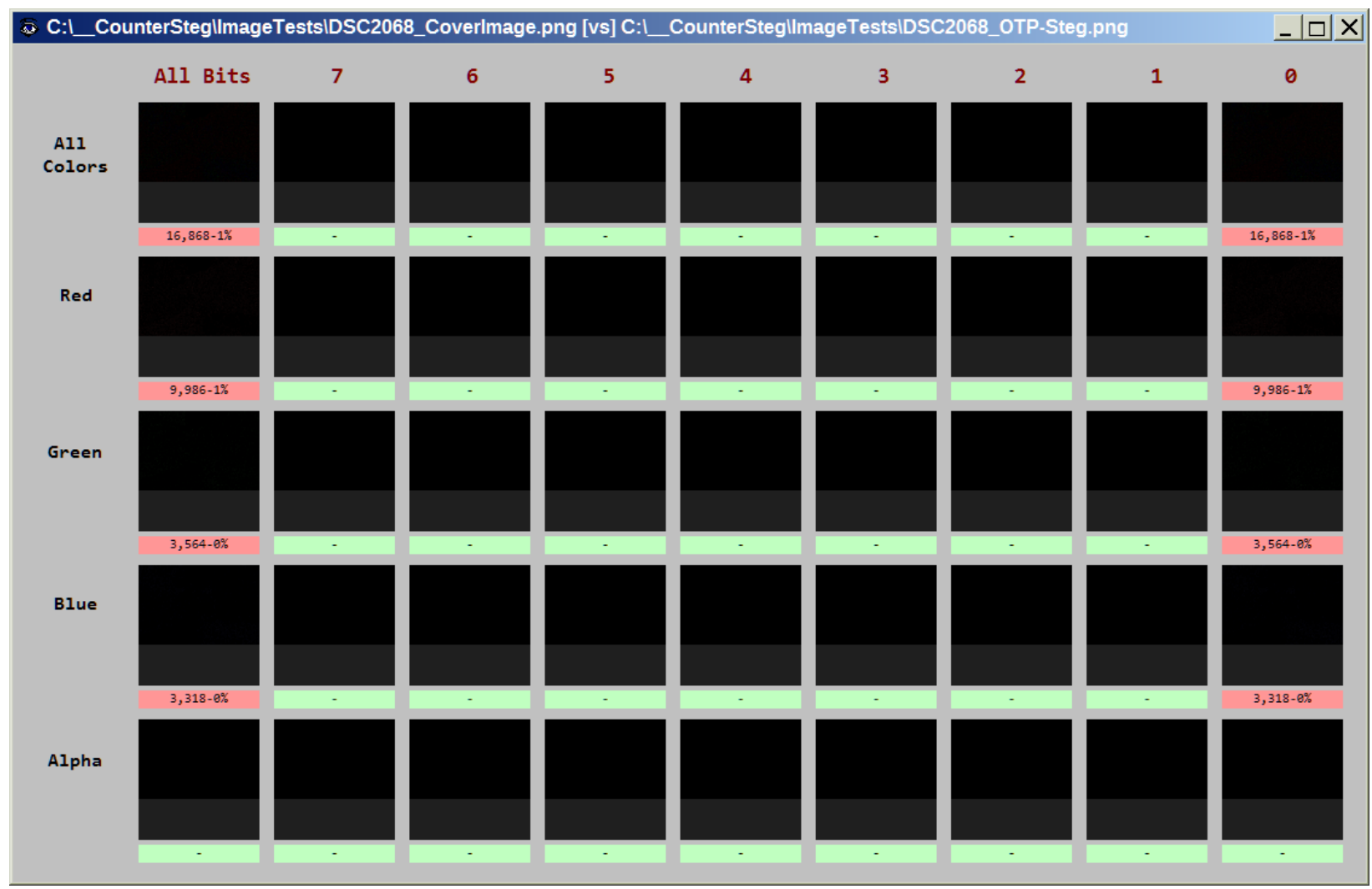

Figure 33. OTP-Steg image comparison. 
Shown below in Figure 34 is the image of the specific LSB values changed, in the three visible colors. Changes are dispersed randomly throughout the image, with respect to noise variation. The saturated sky area is completely avoided. Noticeably, red is much more heavily encoded into them blue and green. This image has a much stronger red component than the other two colors. However, where blue or green are dominant, that is the color encoded into them that respective area. Only one color channel bit per pixel is allowed to be modified.

Figure 34. OTP-Steg LSB image changes. 


\section{SIMILAR IMAGE SEARCH}

To aid in locating images for further investigation, the CounterSteg software includes facilities for similar image searching on all machine drives and directories. The similar image search will identify images of identical pixel dimensions and most significant bit values. The value for search of most significant bit similarity percentage is software selectable. The search window design is shown below as Figure 35.

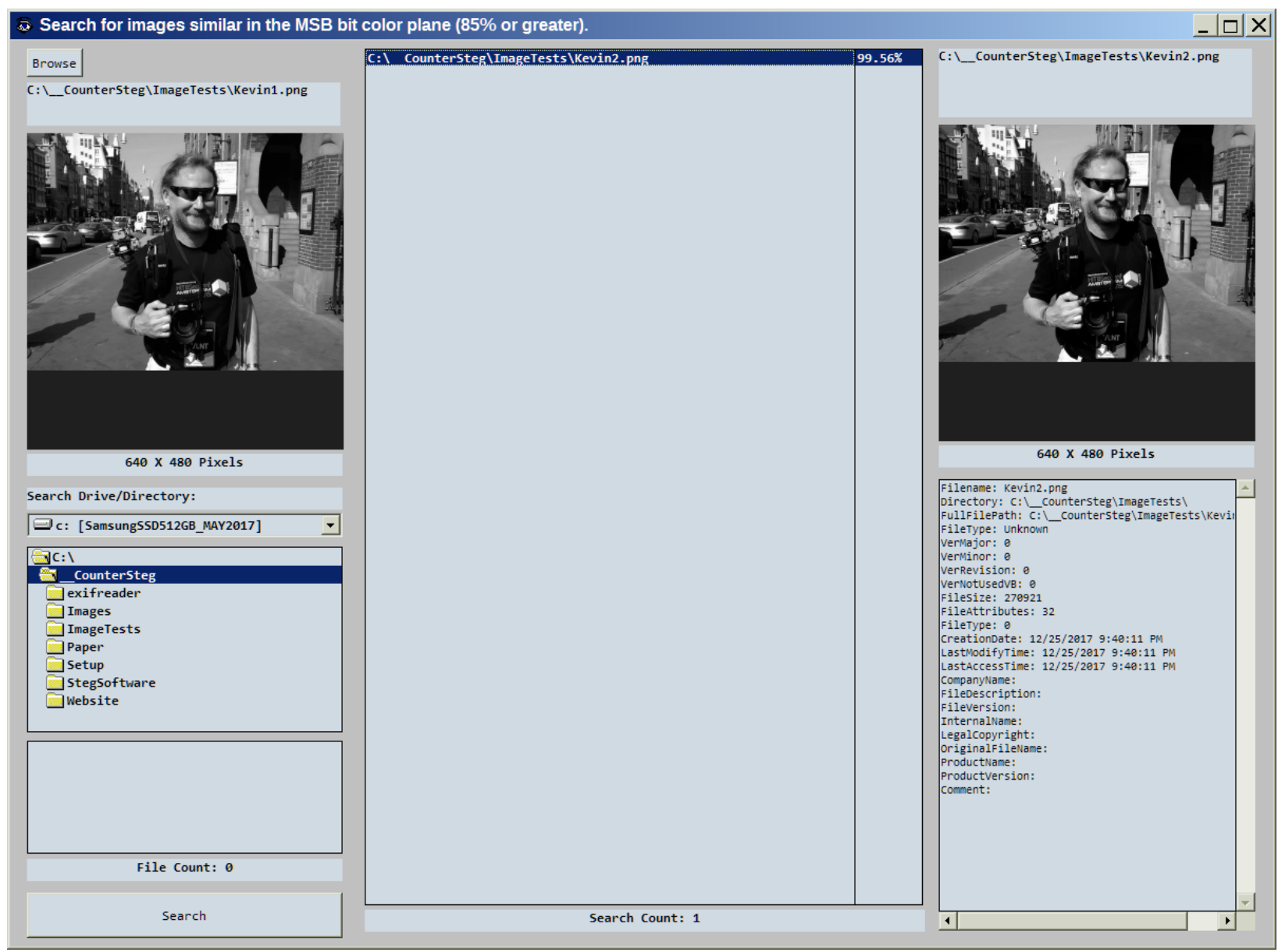

Figure 35. Similar image search (similar in MSB values).

\section{FURTHER RESEARCH}

The authors of this study acknowledge that there is further work to be done in this area. One clear area of research is to automate the positive identification using image LSB comparison by developing algorithms to conduct analyses. The most promising avenue of research in this area appears to be a statistical measure of similarity between pixel color bits above the LSB plane, and difference measures in the LSB plane. In other words, primarily the LSB bits are altered between the image files, preserving the visual appearance, but only altering the data carrying LSB values.

Having such an automated algorithm in hand would greatly assist with the current phenomenon of malware making use of steganography to exfiltrate user and corporate data, such as credit cards, through detection systems designed to thwart such illicit transmissions. Further, web image searches could be conducted by 
the system to check against images currently in transit. Detection of payload carrying images could create a red flag to alert security staff to the fact that data exfiltration may be taking place, or that a malware infestation is receiving command and control messages through payload carrying images.

Also, it may be possible to automate part of the process for similar image searching and retrieval using the Google custom search API facilities.

\section{CONCLUSIONS}

Positively detecting the use of steganography in digital image files currently generally results in an unreliable and inconclusive effort. At least by attempting to actively recover the original image, before pixel bit alteration, it is estimated to make this procedure much more reliable for positive identification by providing investigative software to allow such a comparison efficiently.

Instead of performing statistical analysis to overall produce dubious results, if steganography is suspected, this study posits the investigator would be more effective in simply looking for the original image file for comparison. With comparison software in place, the investigator and assign virtually conclusive attribution. This can be immediately obtained as shown in the numerous examples previously presented in this paper, and useful as evidence in legal proceedings or requests for initial or additional warrants.

\section{REFERENCES}

[1] Anderson, R. \& Petitcolas, F. (1998). On the limits of steganography. IEEE Journal of Selected Areas in Communications, 16 (4), 474-481.

[2] Bashardoust, M., Sulong, G., \& Gerami, P. (2013). Enhanced LSB image steganography method by using knight tour algorithm, vignere encryption and LZW compression. International Journal of Computer Science Issues, 10(2)2, 221-227.

[3] Bhattacharyya, S. (2011). A survey of steganography and steganalysis technique in image, text, audio and video as cover carrier. International Journal of Global Research in Computer Science (UGC Approved Journal), 2(4).

[4] Bureau, Pierre-Marc, and Christian Dietrich. "Hiding in Plain Sight." (2015).

[5] Cabaj, K., Caviglione, L., Mazurczyk, W., Wendzel, S., Woodward, A., \& Zander, S. (2018). The New Threats of Information Hiding: The Road Ahead. IT Professional, 20(3), 31-39.

[6] Chan, C. K., \& Cheng, L. M. (2004). Hiding data in images by simple LSB substitution. Pattern recognition, 37(3), 469-474.

[7] Cheddad, A., Condell, J., Curran, K., \& Mc Kevitt, P. (2010). Digital image steganography: Survey and analysis of current methods. Signal processing, 90(3), 727-752.

[8] Chen, P., Huygens, C., Desmet, L., \& Joosen, W. (2016, May). Advanced or not? A comparative study of the use of anti-debugging and anti-VM techniques in generic and targeted malware. In IFIP International Information Security and Privacy Conference (pp. 323-336). Springer, Cham.

[9] Daryabar, F., Dehghantanha, A., \& Broujerdi, H. G. (2011). Investigation of malware defense and detection techniques. International Journal of Digital Information and Wireless Communications (IJDIWC), $1(3), 645-650$. 
[10] Douiri, S. M., Medeni, M. B. O., Elbernoussi, S., \& Souidi, E. M. (2013). A new steganographic method for grayscale image using graph coloring problem. Appl. Math, 7(2), 521-527.

[11] Easttom, C. (2017). System Forensics, Investigation, and Response, 3rd Edition. Burlington Massachusetts: Jones \& Bartlett.

[12] Easttom, C. (2018). Penetration Testing Fundamentals: A Hands-on Guide to Reliable Security Audits. New York City, New York: Pearson Press

[13] Gangwar, A., \& Shrivastava, V. (2013). Improved RGB-LSB steganography using secret key. International Journal of Computer Trends and Technology, 4(2), 85-89.

[14] Gutte, R. S., Chincholkar, Y. D., \& Lahane, P. U. (2013). Steganography for two and three lsbs using extended substitution algorithm. ICTACT Journal on communication technology, 4(01), 685-690.

[15] Gutub, A., Ankeer, M., Abu-Ghalioun, M., Shaheen, A., \& Alvi, A. (2008). Pixel indicator high capacity technique for RGB image based Steganography.

[16] Gutub, A., Ankeer, M., Abu-Ghalioun, M., Shaheen, A., \& Alvi, A. (2008). Pixel indicator high capacity technique for RGB image based Steganography.

[17] Hamid, N., Yahya, A., Ahmad, R. B., Najim, D., \& Kanaan, L. (2013). Steganography in image files: A survey. Australian Journal of Basic and Applied Sciences, 7(1), 35-55.

[18] He, J., Tang, S., \& Wu, T. (2008, May). An adaptive image steganography based on depth-varying embedding. In Image and Signal Processing, 2008. CISP'08. Congress on (Vol. 5, pp. 660-663). IEEE.

[19] Heidelberg, L. (2016). Steganography in the financial sector (Doctoral dissertation, Utica College).

[20] Hussain, M. \& Hussain, M. (2013). A survey of image steganography techniques. International Journal of Advanced Science and Technology, 54, 113-123.

[21] Jain, Y. K., \& Ahirwal, R. R. (2010). A novel image steganography method with adaptive number of least significant bits modification based on private stego-keys. International Journal of Computer Science and Security, 4(1), 40-49.

[22] Juneja, M., \& Sandhu, P. S. (2009, October). Designing of robust image steganography technique based on LSB insertion and encryption. In Advances in Recent Technologies in Communication and Computing, 2009. ARTCom'09. International Conference on (pp. 302-305). IEEE.

[23] Kamaldeep, (2013). Image steganography techniques in spatial domain, their parameters and analytical techniques: a review article. IJAIR,2 (5), 85-92.

[24] Kekre, H. B., Athawale, A., \& Halarnkar, P. N. (2008). Increased capacity of information hiding in LSBs method for text and image. International Journal of Electrical, Computer and Systems Engineering, 2(4), 246-249.

[25] Lee, Y. K., Bell, G., Huang, S. Y., Wang, R. Z., \& Shyu, S. J. (2009, January). An advanced leastsignificant-bit embedding scheme for steganographic encoding. In Pacific-Rim Symposium on Image and Video Technology (pp. 349-360). Springer, Berlin, Heidelberg. 
[26] Li, B., He, J., Huang, J., \& Shi, Y. Q. (2011). A survey on image steganography and steganalysis. Journal of Information Hiding and Multimedia Signal Processing, 2(2), 142-172.

[27] Lou, D. C., \& Hu, C. H. (2012). LSB steganographic method based on reversible histogram transformation function for resisting statistical steganalysis. Information Sciences, 188, 346-358.

[28] Mazurczyk, W., \& Caviglione, L. (2015). Steganography in modern smartphones and mitigation techniques. IEEE Communications Surveys \& Tutorials, 17(1), 334-357.

[29] Marçal, A. R., \& Pereira, P. R. (2005). A steganographic method for digital images robust to RS steganalysis. In International Conference Image Analysis and Recognition (pp. 1192-1199). Springer, Berlin, Heidelberg.

[30] Martin, A., Sapiro, G., \& Seroussi, G. (2005). Is image steganography natural? IEEE Transactions on Image processing, 14(12), 2040-2050.

[31] Meena, M. K., Kumar, S., \& Gupta, N. (2011). Image Steganography tool using Adaptive Encoding Approach to maximize Image hiding capacity. International Journal of Soft Computing and Engineering (IJSCE), 1(2).

[32] Melanson, M. (2017). Challenges to Law Enforcement in Detection of Steganography (Doctoral dissertation, Utica College).

[33] Mishra, A., Gupta, A., \& Vishwakarma, D. K. (2009, December). Proposal of a new steganographic approach. In Advances in Computing, Control, \& Telecommunication Technologies, 2009. ACT'09. International Conference on (pp. 175-178). IEEE.

[34] Mathkour, H., Assassa, G. M., Al Muharib, A., \& Kiady, I. (2009, April). A novel approach for hiding messages in images. In Signal Acquisition and Processing, 2009. ICSAP 2009. International Conference on (pp. 89-93). IEEE.

[35] Melanson, M. (2017). Challenges to Law Enforcement in Detection of Steganography (Doctoral dissertation, Utica College).

[36] Motameni, H., Norouzi, M., Jahandar, M., \& Hatami, A. (2007). Labeling method in Steganography. World Academy of Science, Engineering and Technology, 24, 349-354.

[37] Parvez, M. T., \& Gutub, A. A. A. (2008, December). RGB intensity based variable-bits image steganography. In Asia-Pacific Services Computing Conference, 2008. APSCC'08. IEEE (pp. 1322-1327). IEEE.

[38] Pevný, T., Kopp, M., Křoustek, J., \& Ker, A. D. (2016). Malicons: Detecting Payload in Favicons. Electronic Imaging, 2016(8), 1-9.

[39] Pharwaha, A. P. S. (2010). Secure data communication using moderate bit substitution for data hiding with three layer security. IE (I) Journal-ET, 91, 45-50.

[40] Priyanka, R., \& Sahoo, P. K. (2016). Scanning Tool for Identification of Image With Malware. IJACTA, 4(1), 170-175. 
[41] Sutherland, I., Davies, G., \& Blyth, A. (2011). Malware and steganography in hard disk firmware. Journal in computer virology, 7(3), 215-219.

[42] Swain, G., \& Lenka, S. K. (2012). LSB array based image steganography technique by exploring the four least significant bits. In Global Trends in Information Systems and Software Applications (pp. 479488). Springer, Berlin, Heidelberg.

[43] Swain, G., \& Lenka, S. K. (2010). A technique for secure communication using message dependent steganography. Special issue of IJCCT, 2(12).

[44] Swain, G., \& Lenka, S. K. (2011, April). Steganography using the twelve square substitution cipher and an index variable. In Electronics Computer Technology (ICECT), 2011 3rd International Conference on (Vol. 3, pp. 84-88). IEEE.

[45] Swain, G., \& Lenka, S. K. (2012). A robust image steganography technique using dynamic embedding with two least significant bits. In Advanced Materials Research (Vol. 403, pp. 835-841). Trans Tech Publications.

[46] Swain, G., \& Lenka, S. K. (2012). A dynamic approach to image steganography using the three least significant bits and extended hill cipher. In Advanced Materials Research (Vol. 403, pp. 842-849). Trans Tech Publications.

[47] Swain, G., \& Lenka, S. K. (2012). A technique for secret communication using a new block cipher with dynamic steganography. International Journal of Security and Its Applications, 6(2), 1-12.

[48] Swain, G., \& Lenka, S. K. (2010, December). A hybrid approach to steganography embedding at darkest and brightest pixels. In Communication and Computational Intelligence (INCOCCI), 2010 International Conference on (pp. 529-534). IEEE.

[49] Walia, E., Jain, P., \& Navdeep, N. (2010). An analysis of LSB \& DCT based steganography. Global Journal of Computer Science and Technology.

[50] Wyke, J. (2015). Breaking the bank (er): Automated configuration data extraction for banking malware.

[51] Younes, M. A. B., \& Jantan, A. (2008). A new steganography approach for images encryption exchange by using the least significant bit insertion. International Journal of Computer Science and Network Security, 8(6), 247-257.

[52] Zhang, H. J., \& Tang, H. J. (2007, August). A novel image steganography algorithm against statistical analysis. In Machine Learning and Cybernetics, 2007 International Conference on (Vol. 7, pp. 3884-3888). IEEE. 